\title{
PRECONDITIONED GRADIENT ITERATIONS FOR THE EIGENPROBLEM OF DEFINITE MATRIX PAIRS*
}

\author{
MARIJA MILOLOŽA PANDUR ${ }^{\dagger}$
}

\begin{abstract}
Preconditioned gradient iterations for large and sparse Hermitian generalized eigenvalue problems $A x=\lambda B x$, with positive definite $B$, are efficient methods for computing a few extremal eigenpairs. In this paper we give a unifying framework of preconditioned gradient iterations for definite generalized eigenvalue problems with indefinite $B$. More precisely, these iterations compute a few eigenvalues closest to the definiteness interval, which can be in the middle of the spectrum, and the corresponding eigenvectors of definite matrix pairs $(A, B)$, that is, pairs having a positive definite linear combination. Sharp convergence theorems for the simplest variants are given. This framework includes an indefinite locally optimal block preconditioned conjugate gradient (LOBPCG) algorithm derived by Kressner, Miloloža Pandur, and Shao [Numer. Algorithms, 66 (2014), pp. 681-703]. We also give a generic algorithm for constructing new "indefinite extensions" of standard (with positive definite $B$ ) eigensolvers. Numerical experiments demonstrate the use of our algorithm for solving a product and a hyperbolic quadratic eigenvalue problem. With excellent preconditioners, the indefinite variant of LOBPCG is the most efficient method. Finally, we derive some ideas on how to use our indefinite eigensolver to compute a few eigenvalues around any spectral gap and the corresponding eigenvectors of definite matrix pairs.
\end{abstract}

Key words. eigenpair, definite matrix pair, definitizing shift, definiteness interval, spectral gap, preconditioned steepest descent/ascent iteration, indefinite LOBPCG

AMS subject classifications. 65F15, 65F08, 65F50

1. Introduction. The generalized eigenvalue problem (GEP) for two given matrices $A, B \in \mathbb{C}^{n \times n}$ is to find scalars $\lambda$ and nonzero vectors $x \in \mathbb{C}^{n}$ such that

$$
A x=\lambda B x .
$$

The pair $(\lambda, x)$ is called an eigenpair, where $\lambda$ is an eigenvalue and $x$ is a corresponding eigenvector. The GEP (1.1) where $A$ and $B$ are both Hermitian or real symmetric occurs in many applications of mathematics. A very important case is when $B$ (and $A$ ) is positive definite, which appears, for example, in the finite element discretization of self-adjoint and elliptic PDE-eigenvalue problems [19]. Another very important case is when $B$ (and $A$ ) is indefinite, but the matrix pair $(A, B)$ is definite, meaning that there exist real scalars $\alpha$ and $\beta$ such that the matrix $\alpha A+\beta B$ is positive definite. This case appears, for example, in mechanics [58] and computational quantum chemistry [3]. Many theoretical properties, such as variational principles, perturbation theory, etc., and eigenvalue solvers for Hermitian matrices have been extended to definite matrix pairs [49, 55, 58].

Suppose $A$ and $B$ are both Hermitian and the pair $(A, B)$ is definite. This excludes singular pairs $(A, B)$, for which $\alpha A+\beta B$ is a singular matrix independent of the choice of $\alpha$ and $\beta$. In this paper, we are interested in solving the partial definite $\operatorname{GEP}(1.1)$, where $B$ (and $A$ ) is indefinite. Even if $A$ and $B$ are both singular, the pair $(A, B)$ can be definite. However, since a definite pair is regular (i.e., it is not a singular pair), the intersection of the nullspaces of such $A$ and $B$ must be trivial [55, Example VI.1.3]. Some of the existing eigenvalue solvers that operate with the indefinite inner product induced by $B$ are indefinite Jacobi algorithms [24, 57], the Rayleigh quotient method [40], and indefinite Lanczos methods [2, 40]. Specifically, in this paper, we are interested in an iterative algorithm that computes a small number of eigenvalues closest to the definiteness interval, see Definition 3.4, and the corresponding eigenvectors.

* Received June 17, 2015. Accepted July 31, 2019. Published online on October 29, 2019. Recommended by Michiel Hochstenbach.

$\dagger$ Josip Juraj Strossmayer University of Osijek, Department of Mathematics, Trg Ljudevita Gaja 6, 31000 Osijek, Croatia (mmiloloz@mathos.hr). 
These eigenvalues are themselves relevant in some applications such as computational quantum chemistry [3,53]. Moreover, as we shall see, every spectral gap can be viewed as the definiteness interval of a related matrix pair. In this way, we devise an algorithm to compute the eigenvalues in an arbitrary interval of the spectrum. The class of algorithms we deal with are preconditioned gradient-type iterations, in single vector or block form, suitable for large and sparse matrices, previously studied for the case in which $A$ and/or $B$ are known to be positive definite; for a survey of preconditioned iterations we refer to [2, 32]. An indefinite variant of the locally optimal block preconditioned conjugate gradient method (the LOBPCG method proposed by Knyazev in [33]) is suggested by Kressner, Miloloža Pandur, and Shao in [38]. In this paper, we propose some new preconditioned eigensolvers [43, Section 3] suitable for a definite matrix pair with indefinite matrices that can be interpreted as a truncated and extended version of the indefinite LOBPCG [38]. For the truncated version of the indefinite locally optimal preconditioned conjugate gradient (LOPCG) method, ${ }^{1}$ which we call the indefinite preconditioned steepest descent/ascent ( $\mathrm{PSD} / \mathrm{A})$, we derive sharp convergence estimates.

This paper is organized as follows. In Section 2, we give a short review of two important preconditioned gradient iterations for a GEP $A x=\lambda B x$ with $A$ positive definite. In Section 3, we present a unifying framework of preconditioned gradient iterations for a definite GEP (1.1) with indefinite $B$ called an indefinite variant of the $(m)$-scheme. Section 4 contains sharp convergence estimates for the simplest variants of the indefinite variant of the $(m)$-scheme. In Section 5, we devise some possibilities of using our algorithm to compute a modest number of eigenvalues around any spectral gap of a definite matrix pair. Numerical examples are given in Section 6. Section 7 contains some concluding remarks.

Notation. The matrix $I_{n}$ denotes the $n \times n$ identity matrix. When the dimension is clear from the context we use simply $I$. We write $A \succ 0(A \succeq 0)$ when $A$ is a Hermitian positive (semi)definite matrix. We also use $A \prec 0(A \preceq 0)$ if $-A \succ 0(-A \succeq 0)$. For a given $A \succ 0$, $\|\cdot\|_{A}$ denotes a matrix norm induced by the vector norm $\|x\|_{A}=\sqrt{x^{H} A x}$. The inertia of a Hermitian matrix $B$ is denoted by $\operatorname{In}(B)$, that is, the ordered triple containing the number of positive, negative, and zero eigenvalues of $B$. A sub- and superscript,+- of some scalar, vector or an iteration name refer to the corresponding $B$-positive and $B$-negative property, respectively.

2. Preliminaries. Let $A, B \in \mathbb{C}^{n \times n}$ be Hermitian and let $A$ be positive definite. We briefly review two known preconditioned gradient iterations for finding the smallest eigenvalue and the corresponding eigenvector of the GEP

$$
A x=\lambda B x .
$$

Let

$$
\rho(x)=\frac{x^{H} A x}{x^{H} B x}, \quad x^{H} B x \neq 0,
$$

denote the Rayleigh quotient associated with the matrix pair $(A, B)$. When $B$ is indefinite, it is convenient $[33,46]$ to consider the dual GEP $B x=\mu A x$ with $\mu=1 / \lambda$ and to find the largest eigenvalue and the corresponding eigenvector. Let $x^{(i)}$ denote the current approximation of the eigenvector corresponding to the smallest eigenvalue of (2.1). For a given Hermitian matrix $T \in \mathbb{C}^{n \times n}$, called the preconditioner (usually, $T=A^{-1}$ or $T \approx A^{-1}$ ), the preconditioned steepest descent (PSD) $[20,29,30]$ iteration takes the following form

$$
x^{(i+1)}=x^{(i)}-\tau^{(i)} T\left(A x^{(i)}-\rho\left(x^{(i)}\right) B x^{(i)}\right),
$$

${ }^{1}$ LOPCG is the method LOBPCG run with block size one, that is, the single vector variant. 
where the scalar iteration parameter $\tau^{(i)}$ is chosen such that $\rho\left(x^{(i+1)}\right)$ is minimized. The LOPCG iteration [31] takes the following form

$$
x^{(i+1)}=x^{(i)}-\gamma^{(i)} x^{(i-1)}-\tau^{(i)} T\left(A x^{(i)}-\rho\left(x^{(i)}\right) B x^{(i)}\right),
$$

where the scalar iteration parameters $\gamma^{(i)}$ and $\tau^{(i)}$ are chosen such that $\rho\left(x^{(i+1)}\right)$ is minimized. The preconditioned residual $w^{(i)}:=\operatorname{Tr}^{(i)}$, where $r^{(i)}:=A x^{(i)}-\rho\left(x^{(i)}\right) B x^{(i)}$, is obtained by solving the linear system $T^{-1} w^{(i)}=r^{(i)}$. In the finite element discretization of a self-adjoint and elliptic partial differential operator, a symmetric positive definite geometric or algebraic multigrid V-cycle preconditioner is commonly used. According to Neymeyr, "typically, symmetric positive definite multigrid preconditioners can be realized with only linearly increasing computational costs (optimal complexity) and, at best, convergence rates can be guaranteed which do not depend on the mesh size" [45, p. 1042]. Also, see [35].

PSD and LOPCG iterations are preconditioned gradient iterations since for the given preconditioner $T$ they use the $T$-gradient of $\rho$. The $T$-gradient reads

$$
\nabla_{T} \rho(x):=T \nabla \rho(x), \quad \text { where } \quad \nabla \rho(x)=\frac{2(A x-\rho(x) B x)}{x^{H} B x} .
$$

The current residual $A x-\rho(x) B x$ is collinear with the gradient $\nabla \rho(x)$, which explains the term "gradient" in gradient iteration. Therefore, the PSD iteration computes a sequence of iterates with decreasing Rayleigh quotients by successive corrections in the negative $T$-gradient direction of the current iterate. LOPCG additionally contains the optimal direction of the previous iterate.

The connection between a three-term recurrence of the LOPCG iteration (2.3) and a threeterm recurrence of the standard linear preconditioned conjugate gradient method (PCG) [50], is pointed out in [33, p. 523]. Numerical experiments in [33, Section 7] demonstrate a similar behavior of the two mentioned methods when the preconditioners and initial approximations are the same for both methods.

REMARK 2.1. The best possible scalar iteration parameters $\tau^{(i)}$ and $\gamma^{(i)}$ in PSD iteration (2.2) and LOPCG iteration (2.3) are not found by using some optimization method. They are given only implicitly. If $x$ is an eigenvector, then so is $\alpha x, \alpha \neq 0$; so what we really want to find is the direction of the next iterate. From (2.2) and (2.3) we see that the next iterate $x^{(i+1)}$ is in the subspace $\operatorname{span}\left[x^{(i)}, w^{(i)}\right]$ and $\operatorname{span}\left[x^{(i)}, x^{(i-1)}, w^{(i)}\right]$, respectively. Therefore, the best approximations for eigenpairs from the subspace are given through the Rayleigh-Ritz procedure [49]. Therefore, $\left(x^{(i+1)}, \rho\left(x^{(i+1)}\right)\right)$ is a Ritz pair of the matrix pair $(A, B)$ with respect to the given subspace. As PSD (LOPCG) aims at minimizing the Rayleigh quotient, $\rho\left(x^{(i+1)}\right)$ is a smaller (the smallest) Ritz value and $x^{(i+1)}$ is the associated Ritz vector.

If we want to find several smallest eigenpairs of GEP (2.1), then we can use the block (or subspace) versions of iterations (2.2) and (2.3): block preconditioned steepest descent (BPSD) and LOBPCG [32, 33], respectively. Standard preconditioned gradient iterations, such as BPSD and LOBPCG, operate with an inner product induced by a positive definite matrix and aim to compute the smallest or largest eigenvalues (i.e., the extremal eigenvalues) and the corresponding eigenvectors. These iterations can be modified in a natural way to compute the eigenvalues around the definiteness interval, which can be in the middle of the spectrum, and the corresponding eigenvectors of a definite matrix pair with indefinite matrices. Therefore, they operate with an indefinite inner product. Indefinite variants of LOBPCG are suggested in [38]. Here we propose the whole class of indefinite variants of preconditioned gradient iterations in Section 3, which includes indefinite LOBPCG methods [38, Algorithms 1 and 2].

3. An indefinite variant of the $(m)$-scheme of preconditioned gradient iterations. In this section, we propose a new class of preconditioned gradient iterations [43, Sections 3.2.2 
and 3.2.3] for definite matrix pairs $(A, B)$ with indefinite $B$. Therefore, we use the indefinite inner product induced by $B$. A vector $x \in \mathbb{C}^{n}$ is called $B$-positive, $B$-negative, and $B$-neutral if $x^{H} B x>0, x^{H} B x<0$, and $x^{H} B x=0$, respectively. A vector $x$ is $B$-normalized if $\left|x^{H} B x\right|=1$. We call vectors $x_{i}$ and $x_{j} B$-orthogonal if $x_{i}^{H} B x_{j}=0$ and B-orthonormal if $\left|x_{i}^{H} B x_{j}\right|=\delta_{i j}$, where $\delta_{i j}$ is the Kronecker delta symbol.

Definition 3.1. Let $A, B \in \mathbb{C}^{n \times n}$. A matrix pair $(A, B)$ is called a Hermitian matrix pair if both $A$ and $B$ are Hermitian. A Hermitian matrix pair $(A, B)$ is called positive (negative) definite if there exists a real $\lambda_{0}$ such that $A-\lambda_{0} B$ is positive (negative) definite.

REMARK 3.2. Recall that a Hermitian matrix pair $(A, B)$ is definite (e.g., [40]) if there exist real constants $\alpha, \beta$ such that $\alpha A+\beta B$ is a positive definite matrix. In this case, if $\alpha \neq 0$, then $(A, B)$ is a positive or negative definite pair with $\lambda_{0}=-\frac{\beta}{\alpha}$; if $\alpha=0$, then $\beta \neq 0$, and $B$ is either a positive or negative definite matrix. If the pair $(A, \stackrel{\alpha}{B})$ is positive definite, then the pair $(-A, B)$ is negative definite, so from now on we deal only with positive definite matrix pairs.

A definite matrix pair can be diagonalized by a congruence transformation. The following theorem reveals the natural structure of a positive definite matrix pair.

THEOREM $3.3([39,42])$. Let $(A, B)$ be a positive definite matrix pair of order $n$ such that $B$ has inertia $\operatorname{In}(B)=\left(n_{+}, n_{-}, n_{0}\right)$.

i) There exists a nonsingular $W$ such that

$$
W^{H} A W=\left[\begin{array}{ccc}
\Lambda_{+} & & \\
& -\Lambda_{-} & \\
& & I_{n_{0}}
\end{array}\right], \quad W^{H} B W=\left[\begin{array}{lll}
I_{n_{+}} & & \\
& -I_{n_{-}} & \\
& & 0_{n_{0}}
\end{array}\right],
$$

where $\Lambda_{+}:=\operatorname{diag}\left(\lambda_{1}^{+}, \ldots, \lambda_{n_{+}}^{+}\right), \Lambda_{-}:=\operatorname{diag}\left(\lambda_{1}^{-}, \ldots, \lambda_{n_{-}}^{-}\right)$with

$$
\lambda_{n_{-}}^{-} \leq \cdots \leq \lambda_{1}^{-}<\lambda_{1}^{+} \leq \cdots \leq \lambda_{n_{+}}^{+} .
$$

ii) The matrix pair $(A, B)$ has only real finite eigenvalues and the number of finite eigenvalues is $\operatorname{rank}(B)=n_{+}+n_{-}$. Each eigenvalue $\lambda_{j}^{+}, \lambda_{j}^{-}$has an eigenvector $x$ that satisfies $x^{H} B x=1$ and $x^{H} B x=-1$, respectively.

iii) The matrix $A-\lambda_{0} B$ is positive definite for every $\lambda_{0} \in\left(\lambda_{1}^{-}, \lambda_{1}^{+}\right)$and nowhere else.

Note that the definiteness of the pair $(A, B)$ precludes defective eigenvalues. Therefore, since every eigenvalue $\lambda_{j}^{+}$has a $B$-positive eigenvector, $\lambda_{j}^{+}$is called a $B$-positive eigenvalue. Similar holds for $\lambda_{j}^{-}$. Any eigenvector $x$ belonging to a finite eigenvalue of $(A, B)$ cannot be $B$-neutral, so we can always $B$-normalize $x$. Eigenvectors belonging to different eigenvalues are $B$-orthogonal. Theorem 3.3 justifies the following definition.

Definition 3.4. Let $A-\lambda_{0} B$ be positive definite, where $(A, B)$ is a given Hermitian pair. The set of all such $\lambda_{0}$ is an open interval called the definiteness interval [58], and every such $\lambda_{0}$ is called a definitizing shift.

The following lemma guarantees a basic property of our algorithm.

Lemma 3.5 ([38, Section 2]). Let $B \in \mathbb{C}^{n \times n}$ be Hermitian, and consider a partitioned matrix $U=[X, Y] \in \mathbb{C}^{n \times p}$. Moreover, let $\operatorname{In}\left(X^{H} B X\right)=:\left(k_{+}, k_{-}, k_{0}\right)$ and also $\operatorname{In}\left(U^{H} B U\right)=:\left(p_{+}, p_{-}, p_{0}\right)$. Then

$$
k_{+} \leq p_{+}, \quad k_{-} \leq p_{-} .
$$

Now we briefly present the theoretical background for our algorithm [38, Section 2]. Let $A, B \in \mathbb{C}^{n \times n}$ be Hermitian such that $B$ has inertia $\operatorname{In}(B)=\left(n_{+}, n_{-}, n_{0}\right)$ and let $(A, B)$ be a positive definite matrix pair with finite eigenvalues (3.1) and infinite eigenvalues

$$
\lambda_{i}^{\infty}:=\infty \text { for } i=1, \ldots, n_{0} .
$$




\section{ETNA}

Kent State University and

Johann Radon Institute (RICAM)

Now, the definiteness interval equals $\left(\lambda_{1}^{-}, \lambda_{1}^{+}\right)$. Let $U \in \mathbb{C}^{n \times p}$ have full column rank. Then the projected matrix pair $\left(U^{H} A U, U^{H} B U\right)$ is also positive definite [38, Theorem 2.3]; hence its finite eigenvalues are real and can be ordered as follows:

$$
\theta_{p_{-}}^{-} \leq \cdots \leq \theta_{1}^{-}<\theta_{1}^{+} \leq \cdots \leq \theta_{p_{+}}^{+}
$$

with $\operatorname{In}\left(U^{H} B U\right)=\left(p_{+}, p_{-}, p_{0}\right)$. The eigenvalue interlacing properties [38, Theorem 2.3] hold

$$
\begin{aligned}
& \lambda_{i}^{+} \leq \theta_{i}^{+} \leq \lambda_{i+n-p}^{+} \quad \text { for } \quad i=1, \ldots, p_{+}, \\
& \lambda_{j}^{-} \geq \theta_{j}^{-} \geq \lambda_{j+n-p}^{-} \quad \text { for } \quad j=1, \ldots, p_{-},
\end{aligned}
$$

where we formally set $\lambda_{i}^{+}=\infty$ for $i>n_{+}$and $\lambda_{j}^{-}=-\infty$ for $j>n_{-}$. Let

$$
J_{k}:=\left[\begin{array}{ll}
I_{k_{+}} & \\
& -I_{k_{-}}
\end{array}\right]
$$

for some integers $k_{+}$and $k_{-}$satisfying $\left(k_{+}, k_{-}, 0\right) \leq \operatorname{In}(B)$, where the inequality is understood elementwise. Now assume $\left(k_{+}, k_{-}, 0\right) \leq \operatorname{In}\left(U^{H} B U\right)$. Then applying the trace minimization principle $[37,42]$ to $(A, B)$ and $\left(U^{H} A U, U^{H} B U\right)$, and using the eigenvalue interlacing properties (3.3) we have

$$
\begin{aligned}
& \min _{\substack{X \in \mathbb{C}^{n \times k} \\
X^{H} B X=J_{k}}} \operatorname{trace}\left(X^{H} A X\right)=\sum_{i=1}^{k_{+}} \lambda_{i}^{+}-\sum_{j=1}^{k_{-}} \lambda_{j}^{-} \\
& \leq \sum_{i=1}^{k_{+}} \theta_{i}^{+}-\sum_{j=1}^{k_{-}} \theta_{j}^{-} \\
& =\underset{\substack{Y \in \mathbb{C}^{p \times k} \\
Y^{H}\left(U^{H} B U\right) Y=J_{k}}}{\operatorname{trace}\left(Y^{H}\left(U^{H} A U\right) Y\right),}
\end{aligned}
$$

with equality if and only if $U$ is spanned by the eigenvectors of the pair $(A, B)$ belonging to $\lambda_{1}^{+}, \ldots, \lambda_{k_{+}}^{+}$and $\lambda_{1}^{-}, \ldots, \lambda_{k_{-}}^{-}$. These eigenvectors are columns in the minimizing matrix $X_{\min }$ of the function in (3.4a).

For small given integers $k_{ \pm}$, our aim is to determine the minimum and the minimizing matrix $X_{\min }$ of the function given in (3.4a), that is, to find the $k_{+}$smallest $B$-positive eigenvalues $\lambda_{1}^{+}, \ldots, \lambda_{k_{+}}^{+}$, the $k_{-}$largest $B$-negative eigenvalues $\lambda_{1}^{-}, \ldots, \lambda_{k_{-}}^{-}$, and the corresponding eigenvectors of the positive definite pair $(A, B)$. Considering (3.4), we find approximations of the wanted eigenpairs from the chosen subspace $\mathcal{U}=\operatorname{span} U$ by using the Rayleigh-Ritz procedure. We compute all eigenpairs of the small projected pair $\left(U^{H} A U, U^{H} B U\right)$ and extract only those around its definiteness interval $\theta_{j}^{ \pm}, j=1, \ldots, k_{ \pm}$and the corresponding eigenvectors $y_{j}^{ \pm}, j=1, \ldots, k_{ \pm}$. These eigenvectors are assumed to be normalized such that

$$
Y^{H}\left(U^{H} B U\right) Y=J_{k}, \quad \text { where } \quad Y=\left[y_{k_{+}}^{+}, \ldots, y_{1}^{+}, y_{1}^{-}, \ldots, y_{k_{-}}^{-}\right] .
$$

The Ritz pairs of the matrix pair $(A, B)$ with respect to the subspace $\mathcal{U}$ are then given by $\left(\theta_{j}^{ \pm}, U y_{j}^{ \pm}\right), j=1, \ldots, k_{ \pm}$. Therefore, the matrix $X:=U Y$ of the Ritz vectors has $B$-orthonormal columns.

Now we need to specify a subspace $\mathcal{U}$, which can be chosen in many ways. An indefinite variant of the $(m)$-scheme with one preconditioner is presented in Algorithm 3.1, similarly 


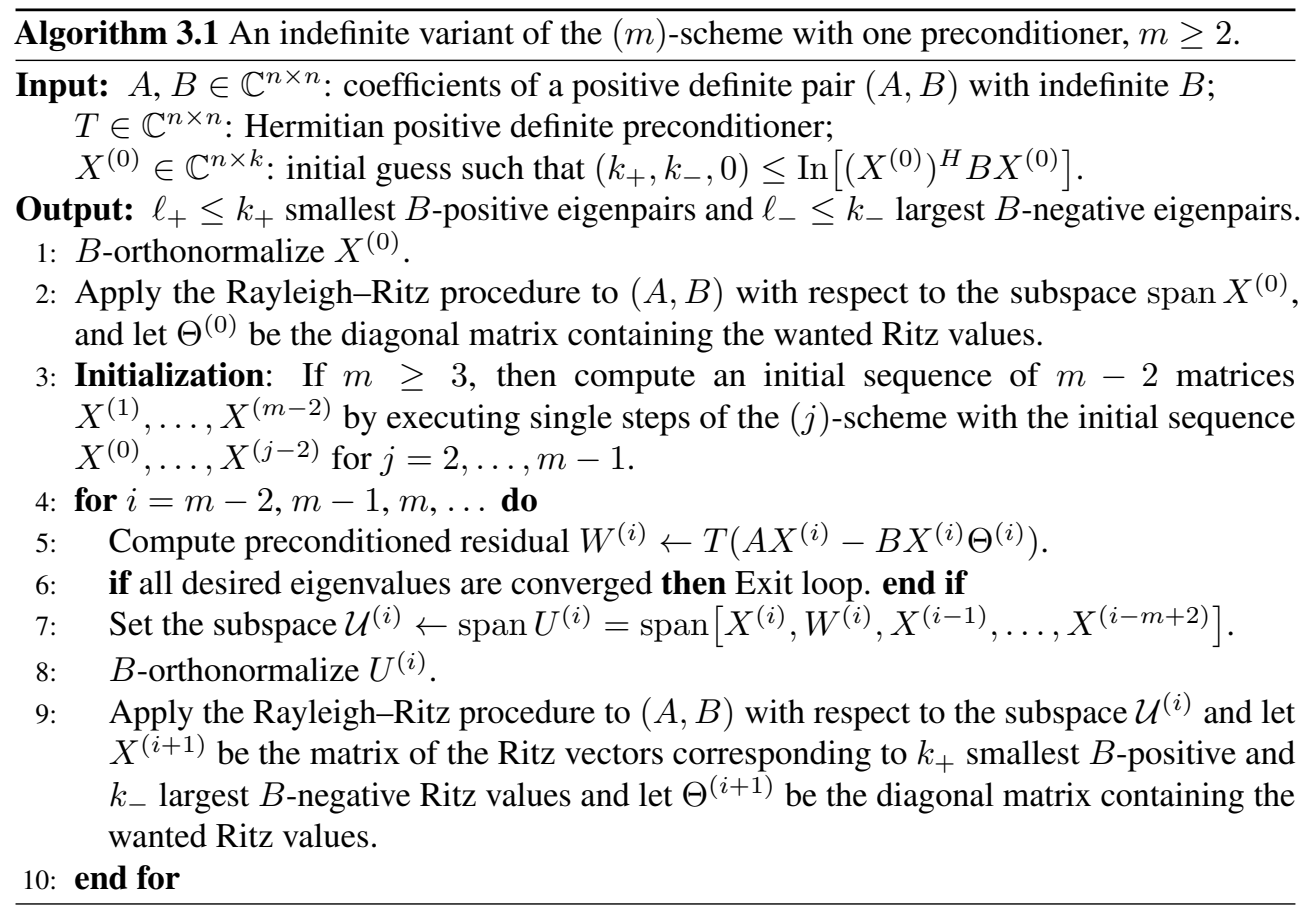

to a unifying framework suggested in $[44,45]$ for a class of preconditioned gradient type eigensolvers for computing the smallest eigenpair of a GEP with real symmetric positive definite matrices. Let

$$
X^{(0)}, \ldots, X^{(m-2)} \in \mathbb{C}^{n \times k}, \quad k=k_{+}+k_{-},
$$

be an initial sequence of matrices of approximations of the wanted eigenvectors with $m \geq 2 \mathrm{a}$ small fixed integer. In the $i$ th $(i \geq m-2)$ iteration of Algorithm 3.1 we consider the subspace

$$
\mathcal{U}^{(i)}=\operatorname{span} U^{(i)}:=\operatorname{span}\left[X^{(i)}, W^{(i)}, X^{(i-1)}, X^{(i-2)}, \ldots, X^{(i-m+2)}\right],
$$

(a matrix $X^{(-j)}$ is a zero matrix) with the preconditioned residual matrix

$$
W^{(i)}:=T \cdot R^{(i)}=T \cdot\left(A X^{(i)}-B X^{(i)} \Theta^{(i)}\right)
$$

for some Hermitian positive definite matrix $T \in \mathbb{C}^{n \times n}$ and

$$
\Theta^{(i)}:=\left(\left(X^{(i)}\right)^{H} B X^{(i)}\right)^{-1}\left(X^{(i)}\right)^{H} A X^{(i)} .
$$

Notice that the dimension of the subspace $\mathcal{U}^{(i)}$ does not exceed $m k$ for all $i=0,1,2, \ldots$.

Every new subspace $\mathcal{U}^{(i)}$ in Algorithm 3.1 contains column vectors of the current iteration matrix $X^{(i)}$ containing Ritz vectors. Therefore, the eigenvalue interlacing properties guarantee $\theta_{j}^{+(i+1)} \leq \theta_{j}^{+(i)}, j=1, \ldots, k_{+}$, and $\theta_{j}^{-(i)} \leq \theta_{j}^{-(i+1)}, j=1, \ldots, k_{-}$, that is, the $B$-positive Ritz values are monotonically decreasing and the $B$-negative Ritz values are monotonically increasing for any preconditioner, not necessarily positive definite. Therefore, our iteration method is trace-reducing and robust with respect to the choice of the preconditioner.

An indefinite LOBPCG method with one preconditioner [38, Algorithm 1] coincides with Algorithm 3.1 with $m=3$.

We now give some remarks on the use and implementation of Algorithm 3.1, similarly to [38, Section 3.1]. The user chooses $m \geq 2$ determining the dimension of the search subspace. 


\section{ETNA}

Kent State University and

Johann Radon Institute (RICAM)

Initial guess. The user needs to give an initial guess $X^{(0)}$ such that

$$
\operatorname{In}\left(\left(X^{(0)}\right)^{H} B X^{(0)}\right)=\left(k_{+}, k_{-}, k_{0}\right) \geq\left(\ell_{+}, \ell_{-}, 0\right)
$$

holds (the inequality is understood elementwise), where $\ell_{+}\left(\ell_{-}\right)$denotes the number of the wanted smallest $B$-positive (largest $B$-negative) eigenpairs. The inequality $k_{ \pm} \leq p_{ \pm}$ for $\operatorname{In}\left(\left(U^{(i)}\right)^{H} B U^{(i)}\right)=\left(p_{+}, p_{-}, p_{0}\right)$ holds for all iterations; this follows by induction from Lemma 3.5. Notice that $p_{+}$and $p_{-}$may vary during the iterative process. Hence, we need precise information about the number of eigenvalues for both types in the projected problem in order to extract the desired Ritz values. This is quite different compared to the standard LOBPCG algorithm, in which the desired Ritz values are always the smallest ones. Fortunately, we have knowledge about $p_{+}$and $p_{-}$since the inertia of $\left(U^{(i)}\right)^{H} B U^{(i)}$ is available as a byproduct of the $B$-orthogonalization procedure; see below.

When $B$ has a particular structure, as in many applications, choosing such an initial guess is straightforward; see Examples 6.1-6.3.

We allow $X^{(i)}$ to have more than $\ell_{+} B$-positive and more than $\ell_{-} B$-negative columns, which can be useful when we have a cluster of eigenvalues. In this way, the dimension of the search subspace is bigger, which can lead to faster convergence.

Initialization. The current subspace $\mathcal{U}^{(i)}$ depends on the span of $m-2$ matrices of the previous Ritz vectors. Therefore, if $m \geq 3$, we need to compute these matrices before proceeding with the iteration. For example, if $m=4$, we need to compute an initial sequence of two matrices $X^{(1)}, X^{(2)}$ by executing single steps of the indefinite variant of the $(j)$-scheme with the initial sequence $X^{(0)}, X^{(j-2)}$ for $j=2,3$. This means that we execute only the first iteration step $(i=0)$ of the (2)-scheme to get $X^{(1)}$ from $\operatorname{span}\left[X^{(0)}, W^{(0)}\right]$. Then, we execute only the first iteration step $(i=1)$ of the (3)-scheme to get $X^{(2)}$ from $\operatorname{span}\left[X^{(1)}, W^{(1)}, X^{(0)}\right]$. Finally, we can proceed with the iteration in the (4)-scheme, where in the first iteration step $(i=2)$ we have $\operatorname{span}\left[X^{(2)}, W^{(2)}, X^{(1)}, X^{(0)}\right]$.

Choosing the basis. The natural basis $\left[X^{(i)}, W^{(i)}, X^{(i-1)}\right]$ for $\mathcal{U}^{(i)}$ in the indefinite LOBPCG method (as in the standard LOBPCG method) is ill-conditioned. To improve numerical stability a new basis is chosen [33,38]: the matrix $X^{(i-1)}$ is replaced by the matrix $P^{(i)}$. The columns of the matrix $P^{(i)}$ are given as an implicit difference of the corresponding columns of the matrices $X^{(i)}$ and $X^{(i-1)}$. More precisely, let the $3 k \times k$ matrix $Y^{(i+1)}$ containing the desired eigenvectors of the projected pair $\left(\left(U^{(i)}\right)^{H} A U^{(i)},\left(U^{(i)}\right)^{H} B U^{(i)}\right)$ be partitioned as

$$
Y^{(i+1)}=\left[\begin{array}{l}
Y_{1}^{(i+1)} \\
Y_{2}^{(i+1)}
\end{array}\right], \quad Y_{1}^{(i+1)} \in \mathbb{C}^{k \times k}, \quad Y_{2}^{(i+1)} \in \mathbb{C}^{2 k \times k} .
$$

$\operatorname{Update}\left(P^{(0)} \leftarrow[]\right)$

$$
P^{(i+1)} \leftarrow\left[W^{(i)}, X^{(i-1)}\right] Y_{2}^{(i+1)}, \quad X^{(i+1)} \leftarrow X^{(i)} Y_{1}^{(i+1)}+P^{(i+1)} .
$$

Since $P^{(i+1)}=X^{(i+1)}-X^{(i)} Y_{1}^{(i+1)}$, then in the indefinite variant of the $(m)$-scheme we use the new basis $\left[X^{(i)}, W^{(i)}, P^{(i)}, P^{(i-1)}, \ldots, P^{(i-m+3)}\right]$ instead of the natural basis $\left[X^{(i)}, W^{(i)}, X^{(i-1)}, X^{(i-2)}, \ldots, X^{(i-m+2)}\right]$.

B-orthonormality. The Ritz pairs extracted from the subspace $\mathcal{U}^{(i)}$ do not depend on the choice of the basis. For the standard LOBPCG method it has been observed that choosing an orthonormal basis leads to improved numerical stability [17, 25]. In [38], the authors made a similar observation when choosing a $B$-orthonormal basis in the indefinite LOBPCG method. 
We prefer $B$-orthonormalization rather than standard orthonormalization (in the Euclidean inner product) in our algorithm for the following reasons. The first reason is the natural structure of the GEP we are interested in: since the eigenvectors corresponding to different eigenvalues of a definite matrix pair are $B$-orthogonal, we would like the approximations of the wanted eigenvectors (in our algorithm, these are Ritz vectors) to be $B$-orthogonal as well. Furthermore, we want to solve the trace minimization problem (3.4), where we have the $B$-orthonormality property $X^{H} B X=\operatorname{diag}( \pm 1)$. Once the basis has been constructed, we form the projected pair $\left(\left(U^{(i)}\right)^{H} A U^{(i)},\left(U^{(i)}\right)^{H} B U^{(i)}\right)$, where, in general, $\left(U^{(i)}\right)^{H} A U^{(i)}$ is a full matrix, and in theory $\left(U^{(i)}\right)^{H} B U^{(i)}=\operatorname{diag}( \pm 1)$. Therefore, we can apply the $J$-Jacobi method of Veselić [57] to the small projected pair.

The $B$-orthonormalization process [38, Section 3.1] needs to be implemented carefully to avoid numerical instability. Notice that this process in not always possible due to the existence of $B$-neutral vectors. Further, for a vector close to a $B$-neutral vector, forcing $B$-normalization will lead to a large growth factor. To avoid that, we can use a preprocessing step [38, Section 3.1] and drop the problematic vectors from the basis; see also the discussion in [17, Section 4.1] for the standard LOBPCG. By Lemma 3.5, when orthogonalizing $U^{(i)}$ from (3.5) with $\left(X^{(i)}\right)^{H} B X^{(i)}=\operatorname{diag}( \pm 1)$, the output $U_{\text {drop }}^{(i)}$ can be chosen of the form $\left[X^{(i)}, Z^{(i)}\right]$ (con-

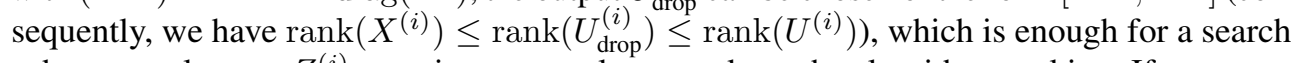
subspace as long as $Z^{(i)}$ contains some columns to keep the algorithm working. If too many columns must be dropped, the basis can be padded with randomly generated $B$-orthogonalized columns.

Deflation. A Ritz pair is deflated after it has converged to the desired accuracy. In our algorithm, we use a "deflate from the middle" strategy: a Ritz value $\theta_{j}^{ \pm}$(here we drop the superscript $(i)$ for clarity) is deflatable if and only if all Ritz values $\theta_{i}^{ \pm}$, with $1 \leq i \leq j-1$, are deflatable and ${ }^{2}$

$$
\left\|r_{j}^{ \pm}\right\|_{2}:=\left\|A x_{j}^{ \pm}-\theta_{j}^{ \pm} B x_{j}^{ \pm}\right\|_{2} \leq \text { tol } \cdot\left|\theta_{j}^{ \pm}\right|\|B\|_{2}\left\|x_{j}^{ \pm}\right\|_{2},
$$

where $x_{j}^{ \pm}:=U y_{j}^{ \pm}$is the corresponding Ritz vector and tol is a tolerance specified by the user. Deflated Ritz vectors, as in the standard LOBPCG method [34], do not participate in the computation of $W^{(i)}$ or $P^{(j)}, j=i-m+3, \ldots, i$, but they still need to part of the $B$-orthonormalization process to avoid repeated convergence to the same eigenvalue. The bound (3.6) is used in a backward error analysis of approximate eigenpairs [38, Section 3.4]. For large sparse matrices an estimate of the 2-norm is used. We refer to [17, Section 4] for this issue. The 1-norm of the matrix can also be used; see, e.g., [41] for a hyperbolic quadratic eigenvalue problem.

Preconditioner. The preconditioned residuals (here we drop the superscript $(i)$ for clarity) $w_{j}^{ \pm}:=T r_{j}^{ \pm}$are obtained by solving linear systems $T^{-1} w_{j}^{ \pm}=r_{j}^{ \pm}$for $j=1, \ldots, k_{ \pm}$. Usually, these systems are solved only approximately by some iterative method such as the linear conjugate gradient method (CG) [16] with a low drop tolerance. Ideally, one application of a preconditioner to a residual vector has the same costs as one matrix-vector product. We use a Hermitian positive definite preconditioner $T$ for three reasons. Since we want to compute the interior eigenvalues around the definiteness interval, we use a definitizing shift $\lambda_{0}$ for which the matrix $A-\lambda_{0} B$ is positive definite. Therefore we use a Hermitian positive definite preconditioner $T=\left(A-\lambda_{0} B\right)^{-1}$ or $T \approx\left(A-\lambda_{0} B\right)^{-1}$. The second reason is the efficiency of a positive definite preconditioner such as a symmetric positive definite multigrid

\footnotetext{
${ }^{2}$ Although we use a superscript $+(-)$ in a residual $r_{j_{+}}^{+}\left(r_{j}^{-}\right)$, this does not imply that $r_{j}^{+}\left(r_{j}^{-}\right)$is $B$-positive (B-negative). Similar holds for a preconditioned residual $w_{j}^{ \pm}$.
} 


\section{ETNA}

Kent State University and

Johann Radon Institute (RICAM)

preconditioner used in the discretization of an elliptic PDE-eigenproblem. The third reason lies in the fact that the convergence analysis (see Section 4.1) is given only for a symmetric positive definite preconditioner. Since our Algorithm 3.1 is robust with the choice of the preconditioner, we can even choose the shift $\lambda_{0}$ outside of the definiteness interval, which results in an indefinite preconditioner [33], [38, Example 5.2].

TABLE 3.1

An indefinite variant of the $(m)$-scheme for $m=2$ and $m=3$.

\begin{tabular}{llll|llll}
$m$ & $k_{-}$ & $k_{+}$ & Eigensolver & $m$ & $k_{-}$ & $k_{+}$ & Eigensolver \\
\hline 2 & 1 & 0 & PSA $^{-}$ & 2 & 0 & 1 & PSD $^{+}$ \\
2 & $>1$ & 0 & BPSA $^{-}$ & 2 & 0 & $>1$ & BPSD $^{+}$ \\
3 & 1 & 0 & LOPCG $^{-}$ & 3 & 0 & 1 & LOPCG $^{+}$ \\
3 & $>1$ & 0 & LOBPCG $^{-}$ & 3 & 0 & $>1$ & LOBPCG $^{+}$ \\
2 & 1 & 1 & indefinite PSD/A & 3 & 1 & 1 & indefinite LOPCG \\
2 & $>1$ & $>1$ & indefinite BPSD/A & 3 & $>1$ & $>1$ & indefinite LOBPCG
\end{tabular}

In Table 3.1, we appoint an eigensolver for $m=2$ and $m=3$ from our algorithm. The names of our methods point to a strong relationship with existing methods for the partial GEP for a matrix pair $(A, B)$ with positive definite $B$. The abbreviation BPSA stands for the block (or subspace) preconditioned steepest ascent iteration. In our methods, superscripts + and mean that iterations operate not on the whole Euclidean space, but only on the $B$-positive and $B$-negative subsets, respectively.

During the past few years several variants of extended (B)PSD/A and LO(B)PCG type methods were proposed for nonlinear Hermitian eigenvalue problems with variational characterizations $[7,41,56]$. The mentioned eigenvalue problems include definite matrix pairs. A so-called interval of definite type: positive ${ }^{3}$ or negative [56, Definition 2.1, Proposition 2.4] is chosen. Then, in a single vector version, the algorithms in [7, 41, 56] compute the smallest (or largest) eigenvalue in that interval and the corresponding eigenvector. In block versions, the algorithms compute a few of the smallest (or largest) eigenvalues in that interval and the corresponding eigenvectors. Our Algorithm 3.1 with $k_{ \pm} \geq 1$ simultaneously computes the eigenvalues on both sides of the definiteness interval and the corresponding eigenvectors of a positive definite matrix pair. Algorithm 3.1 with $m=2,3$ and $k_{+} \geq 1$ and $k_{-}=0$, or $k_{+}=0$ and $k_{-} \geq 1$, coincides with the corresponding algorithms in $[7,56]$. For example, our LOPCG $^{+}$with $k_{+}=1$ coincides with [7, Algorithm 2.1, LOCG $(1,2)$, with a fixed preconditioner and $F(\lambda)=\lambda B-A$ ]. The mentioned extended variants of (B)PSD/A and LO(B)PCG type methods in [7, 41], as discussed in [21, 51, 52], mean that, for example in PSD (2.2), the search subspace $\operatorname{span}\left[x^{(i)}, T\left(A-\rho\left(x^{(i)}\right) B\right) x^{(i)}\right]$ is replaced with the $m$ th order Krylov subspace (usually with a small $m$; here $\rho^{(i)}:=\rho\left(x^{(i)}\right)$ )

$$
\mathcal{K}_{m}\left(T\left(A-\rho^{(i)} B\right) ; x^{(i)}\right):=\operatorname{span}\left[x^{(i)}, T\left(A-\rho^{(i)} B\right) x^{(i)}, \ldots,\left(T\left(A-\rho^{(i)} B\right)\right)^{m-1} x^{(i)}\right] .
$$

The global convergence of the method using $\mathcal{K}_{m}\left(T\left(A-\rho^{(i)} B\right) ; x^{(i)}\right)$ as a search subspace with $T=I$ to some eigenvalue of a definite matrix pair $(A, B)$ with $B \succ 0$ has been proven in [21, Theorem 1]. The global convergence of the LOPCG type method to the extremal eigenpair, for a general Hermitian matrix polynomial with variational characterizations, without any assumption on a preconditioner (including variable preconditioners), is proven in [7, Theorem 2.1]. In particular, an asymptotic estimate for our indefinite $\mathrm{LOPCG}^{+}$method with a fixed positive definite preconditioner is given in [7, Theorem 3.1, $\operatorname{LOCG}(1,2)]$.

\footnotetext{
${ }^{3}$ E.g., $\left(\lambda_{0}, \infty\right)$ is an interval of positive type of a positive definite matrix pair, where $\lambda_{0}$ is a definitizing shift.
} 
It is natural to expect, as confirmed by our convergence analysis from Section 4.1, that the eigenvalues closest to the definitizing shift $\lambda_{0}$ converge first. Since we want to compute the eigenvalues around the definiteness interval, to be more efficient, we can use two definitizing shifts, i.e., $\lambda_{0}^{+}$close to $\lambda_{1}^{+}$and $\lambda_{0}^{-}$close to $\lambda_{1}^{-}$. Therefore, we can use two preconditioners $T_{+} \approx\left(A-\lambda_{0}^{+} B\right)^{-1}$ and $T_{-} \approx\left(A-\lambda_{0}^{-} B\right)^{-1}$, exactly as in [38, Algorithm 2], and get an indefinite variant of the $(m)$-scheme with two preconditioners. To preserve the dimension of the search subspace, we use

$$
\mathcal{U}^{(i)}:=\operatorname{span}\left[X^{(i)}, T_{+} \cdot R_{+}^{(i)}, T_{-} \cdot R_{-}^{(i)}, X^{(i-1)}, \ldots, X^{(i-m+2)}\right]
$$

as a new subspace, where we split the residual matrix $R^{(i)}$ into two parts $R_{+}^{(i)}$ and $R_{-}^{(i)}$ associated with $B$-positive Ritz values and $B$-negative Ritz values, respectively.

4. The simplest variants: PSD $^{+}$and PSA $^{-}$. In this section, we consider the simplest variants in Algorithm 3.1, namely $\mathrm{PSD}^{+}$and $\mathrm{PSA}^{-}$. When $B$ is positive definite, then the PSD (PSA) iteration aims to compute the smallest (the largest) eigenvalue and the corresponding eigenvector of a matrix pair $(A, B)$. If $B \succ 0$, then PSD $=\mathrm{PSD}^{+}$; if $B \prec 0$, then PSA $=\mathrm{PSA}^{-}$. Therefore, when $B$ is indefinite, as in our case, the $\mathrm{PSD}^{+}\left(\mathrm{PSA}^{-}\right)$ iteration aims to compute the smallest (the largest) $B$-positive ( $B$-negative) eigenvalue and the corresponding eigenvector. To give convergence theorems for our methods $\mathrm{PSD}^{+}$and PSA $^{-}$[43, Section 3.2.2], we use the convergence theorem [46] derived for the PSD iteration that is proven only for real symmetric matrices. Therefore, only in this section we assume that $A$ and $B$ are real and symmetric.

Let

$$
\rho(x)=\frac{x^{T} A x}{x^{T} B x}, \quad x^{T} B x \neq 0,
$$

denote the Rayleigh quotient associated with a positive definite matrix pair $(A, B)$. The single vector iteration $\mathrm{PSD}^{+}$(replacing $X^{(i)}, W^{(i)}$, and $X^{(i+1)}$ by $x_{+}, w_{+}$, and $x_{+}^{\prime}$, respectively) uses the subspace $\mathcal{U}^{(i)}=\operatorname{span}\left[x_{+}, w_{+}\right]$in the $i$ th iteration step. ${ }^{4}$ A Ritz pair of $(A, B)$ with respect to the subspace $\mathcal{U}^{(i)}$ is $\left(x_{+}^{\prime}, \rho\left(x_{+}^{\prime}\right)\right)$, where $\rho\left(x_{+}^{\prime}\right)$ is the smaller $B$-positive Ritz value. Similar holds for the $\mathrm{PSA}^{-}$iteration.

REMARK 4.1. The indefinite PSD/A iteration combines the $\mathrm{PSD}^{+}$and the PSA ${ }^{-}$iterations into one iteration method by using the subspace $\mathcal{U}^{(i)}=\operatorname{span}\left[X^{(i)}, W^{(i)}\right]$, where $X^{(i)}=\left[x_{+}, x_{-}\right]$contains the current approximations of the wanted eigenvectors corresponding to $\lambda_{1}^{+}$and $\lambda_{1}^{-}$. The subspace $\mathcal{U}^{(i)}$ in Algorithm 3.1 with $m=3$ and $k_{ \pm}=1$, that is, the indefinite LOPCG iteration, additionally contains the column space of the previous iterate. Therefore, the indefinite PSD/A iteration can be interpreted as a truncated version of the indefinite LOPCG iteration. Similarly, higher order schemes in Algorithm 3.1 with $m>3$ and $k_{ \pm}=1$, can be interpreted as an extended version of the indefinite LOPCG method. Similar holds for the block versions.

4.1. Convergence theorems for $\mathbf{P S D}^{+}$and $\mathbf{P S A}^{-}$. A sharp convergence theorem for the standard PSD iteration applied to a real symmetric matrix pair $(\widetilde{A}, B)$, where $\widetilde{A}$ is positive definite, is given in [46], using the dual matrix pair $(B, \widetilde{A})$. To give corresponding convergence theorems for eigenvalues around the definiteness interval of a positive definite matrix pair $(A, B)$ with indefinite $B$ computed by $\mathrm{PSD}^{+}$and $\mathrm{PSA}^{-}$, we need to present them as the extremal eigenvalues of some auxiliary matrix pair.

\footnotetext{
${ }^{4}$ If $x_{+}$and $w_{+}$are linearly dependent, then the iteration terminates in the current iterate $x_{+}$.
} 
Consider a positive definite matrix pair $(A, B)$ with eigenvalues (3.1) and (3.2). Let $\lambda_{0}$ be a given definitizing shift and let

$$
\widetilde{A}=A-\lambda_{0} B \succ 0, \quad \widetilde{\lambda}=\lambda-\lambda_{0} \neq 0 .
$$

Hence for the eigenproblem

$$
B x=\widetilde{\mu} \widetilde{A} x
$$

the eigenvalues $\{\widetilde{\mu}\}$ are given by

$$
\widetilde{\mu}=1 / \widetilde{\lambda}=1 /\left(\lambda-\lambda_{0}\right)
$$

and arranged in the order:

$$
\begin{gathered}
1 / \widetilde{\lambda}_{1}^{-} \leq \cdots \leq 1 / \widetilde{\lambda}_{n_{-}}^{-}<0=1 / \widetilde{\lambda}_{1}^{\infty}=\cdots=1 / \widetilde{\lambda}_{n_{0}}^{\infty}<1 / \widetilde{\lambda}_{n_{+}}^{+} \leq \cdots \leq 1 / \widetilde{\lambda}_{1}^{+} \\
{ }^{\prime \prime} \\
\widetilde{\mu}_{1}^{-} \leq \cdots \leq \widetilde{\mu}_{n_{-}}^{\prime \prime}<0=\widetilde{\mu}_{1}^{\infty}=\cdots=\widetilde{\mu}_{n_{0}}^{\infty}<\widetilde{\mu}_{n_{+}}^{\prime} \leq \cdots \leq \widetilde{\mu}_{1}^{+} .
\end{gathered}
$$

Let

$$
\widetilde{\mu}(x)=\frac{1}{\widetilde{\rho}(x)}=\frac{x^{T} B x}{x^{T} \widetilde{A} x}=\frac{1}{\rho(x)-\lambda_{0}} \in \mathbb{R}, \quad x \neq 0,
$$

denote the Rayleigh quotient associated with the matrix pair $(B, \widetilde{A})$ from (4.1). Therefore, the goal of the $\mathrm{PSD}^{+}$iteration is equivalent to computing the largest eigenvalue $\widetilde{\mu}_{1}^{+}$by maximizing $\widetilde{\mu}(x)$ from (4.2), and their corresponding eigenvector. Similar holds for the $\mathrm{PSA}^{-}$iteration. Since we want to compute extremal eigenvalues $\widetilde{\mu}_{1}^{+}$and $\widetilde{\mu}_{1}^{-}$, we can use the $\operatorname{PSD}_{\widetilde{\mu}}$ (4.3) iteration and then apply [46, Theorem 2.2] to the matrix pairs $(B, \widetilde{A})$ and $(-B, \widetilde{A})$ from $(4.1)$, respectively. The transformation of the PSD iteration (2.2) (after multiplication by $\widetilde{\mu}(x)$, replacing $A, \rho, x^{(i+1)}, x^{(i)}$, and $\tau^{(i)}$ by $\widetilde{A}, \widetilde{\rho}, x^{\prime}, x$, and $\tau_{\text {opt }}$, respectively) is

$$
\left(\operatorname{PSD}_{\widetilde{\mu}}\right) \quad \widetilde{\mu}(x) x^{\prime}=\widetilde{\mu}(x) x+\tau_{\mathrm{opt}} T(B x-\widetilde{\mu}(x) \widetilde{A} x)
$$

with the optimal step length

$$
\tau_{\text {opt }}:=\arg \max _{\tau \in \mathbb{R}} \widetilde{\mu}(\widetilde{\mu}(x) x+\tau T(B x-\widetilde{\mu}(x) \widetilde{A} x)) .
$$

For a symmetric positive definite matrix $T$, a preconditioner that approximates the inverse of the positive definite $\widetilde{A}$, one assumes [46, Section 1.1]

$$
\|I-T \widetilde{A}\|_{\widetilde{A}} \leq \gamma, \quad \gamma \in[0,1) .
$$

Neymeyr gives a convergence estimate of the poorest possible convergence of the PSD iteration (2.2) in [46, Theorem 1.2]; the proof of that theorem takes 12 pages. That estimate is sharp in the sense that an initial guess and a preconditioner $T$ satisfying the inequality (4.4) can be chosen such that the bound is attained. [46, Theorem 1.2] guarantees the monotone convergence of a sequence of the Ritz values to some eigenvalue; to the smallest eigenvalue only if the Ritz values have reached the final interval $\left[\lambda_{1}, \lambda_{2}\right)$, but, due to roundoff, in practice that sequence almost surely converge to the smallest eigenvalue. A similar convergence theorem for the $\mathrm{PSD}^{+}$and $\mathrm{PSA}^{-}$iteration is as follows: 
THEOREM 4.2. Let $x_{+} \in \mathbb{R}^{n}$ and $x_{+}^{\prime}$ be the PSD ${ }^{+}$iterate. The preconditioner $T$ is assumed to satisfy (4.4). If $\lambda_{i}^{+} \leq \rho\left(x_{+}\right)<\lambda_{i+1}^{+}, i=1, \ldots, n_{+}-1$, then $\rho\left(x_{+}^{\prime}\right) \leq \rho\left(x_{+}\right)$ and either $\rho\left(x_{+}^{\prime}\right) \leq \lambda_{i}^{+}$or

$$
\begin{aligned}
& \frac{\rho\left(x_{+}^{\prime}\right)-\lambda_{i}^{+}}{\lambda_{i+1}^{+}-\rho\left(x_{+}^{\prime}\right)} \leq \sigma_{i,+}^{2} \frac{\rho\left(x_{+}\right)-\lambda_{i}^{+}}{\lambda_{i+1}^{+}-\rho\left(x_{+}\right)} \\
& \text {with } \quad \sigma_{i,+}:=\frac{\kappa_{i,+}+\gamma\left(2-\kappa_{i,+}\right)}{\left(2-\kappa_{i,+}\right)+\gamma \kappa_{i,+}} \text { and } \kappa_{i,+}:=\frac{\left(\lambda_{i}^{+}-\lambda_{0}\right)\left(\lambda_{1}^{-}-\lambda_{i+1}^{+}\right)}{\left(\lambda_{i+1}^{+}-\lambda_{0}\right)\left(\lambda_{1}^{-}-\lambda_{i}^{+}\right)} .
\end{aligned}
$$

The estimate is sharp and can be attained for $\rho\left(x_{+}\right) \rightarrow \lambda_{i}^{+}$in the three-dimensional invariant subspace associated with the eigenvalues $\lambda_{i}^{+}, \lambda_{i+1}^{+}$, and $\lambda_{1}^{-}$.

Proof. The proof follows from [46, Theorem 2.2] applied to the matrix pair $(B, \widetilde{A})$ from (4.1) with associated substitutions $\sigma \rightarrow \sigma_{i,+}, \mu(x) \rightarrow \widetilde{\mu}\left(x_{+}\right)=1 /\left(\rho\left(x_{+}\right)-\lambda_{0}\right)$, $\left(\mu_{n}, \mu_{i+1}, \mu_{i}, \mu_{1}\right) \rightarrow\left(\widetilde{\mu}_{1}^{-}, \widetilde{\mu}_{i+1}^{+}, \widetilde{\mu}_{i}^{+}, \widetilde{\mu}_{1}^{+}\right), \mu_{j} \rightarrow \widetilde{\mu}_{j}^{ \pm}=1 /\left(\lambda_{j}^{ \pm}-\lambda_{0}\right)$, and $\kappa \rightarrow \kappa_{i,+}$.

THEOREM 4.3. Let $x_{-} \in \mathbb{R}^{n}$ and $x_{-}^{\prime}$ be the PSA ${ }^{-}$iterate. The preconditioner $T$ is assumed to satisfy (4.4). If $\lambda_{i+1}^{-}<\rho\left(x_{-}\right) \leq \lambda_{i}^{-}, i=1, \ldots, n_{-}-1$, then $\rho\left(x_{-}^{\prime}\right) \geq \rho\left(x_{-}\right)$ and either $\rho\left(x_{-}^{\prime}\right) \geq \lambda_{i}^{-}$or

$$
\begin{aligned}
& \frac{\lambda_{i}^{-}-\rho\left(x_{-}^{\prime}\right)}{\rho\left(x_{-}^{\prime}\right)-\lambda_{i+1}^{-}} \leq \sigma_{i,-}^{2} \frac{\lambda_{i}^{-}-\rho\left(x_{-}\right)}{\rho\left(x_{-}\right)-\lambda_{i+1}^{-}} \\
& \text {with } \quad \sigma_{i,-}:=\frac{\kappa_{i,-}+\gamma\left(2-\kappa_{i,-}\right)}{\left(2-\kappa_{i,-}\right)+\gamma \kappa_{i,-}} \text { and } \kappa_{i,-}:=\frac{\left(\lambda_{i}^{-}-\lambda_{0}\right)\left(\lambda_{1}^{+}-\lambda_{i+1}^{-}\right)}{\left(\lambda_{i+1}^{-}-\lambda_{0}\right)\left(\lambda_{1}^{+}-\lambda_{i}^{-}\right)} .
\end{aligned}
$$

The estimate is sharp and can be attained for $\rho\left(x_{-}\right) \rightarrow \lambda_{i}^{-}$in the three-dimensional invariant subspace associated with the eigenvalues $\lambda_{i}^{-}, \lambda_{i+1}^{-}$, and $\lambda_{1}^{+}$.

Proof. The proof follows from [46, Theorem 2.2] applied to the matrix pair $(-B, \widetilde{A})$ from (4.1) with associated substitutions $\sigma \rightarrow \sigma_{i,-}, \mu(x) \rightarrow-\widetilde{\mu}\left(x_{-}\right)=-1 /\left(\rho\left(x_{-}\right)-\lambda_{0}\right)$, $\left(\mu_{n}, \mu_{i+1}, \mu_{i}, \mu_{1}\right) \rightarrow\left(-\widetilde{\mu}_{1}^{+},-\widetilde{\mu}_{i+1}^{-},-\widetilde{\mu}_{i}^{-},-\widetilde{\mu}_{1}^{-}\right), \mu_{j} \rightarrow-\widetilde{\mu}_{j}^{ \pm}=-1 /\left(\lambda_{j}^{ \pm}-\lambda_{0}\right)$, and $\kappa \rightarrow \kappa_{i,-}$.

For $\kappa$ from [46, Theorem 2.2] holds $\kappa \in(0,1)$, which implies $\kappa_{i, \pm} \in(0,1)$. Since $\sigma_{i,+}=\sigma_{i,+}\left(\gamma, \kappa_{i,+}\right)$ is a monotone increasing function in both variables, choosing smaller $\gamma$ (meaning a preconditioner $T$ approaches to the exact inverse of $\widetilde{A}$ ) and/or smaller $\kappa_{i,+}$ will lead to a faster convergence of the $\mathrm{PSD}^{+}$iteration. Similar holds for the $\mathrm{PSA}^{-}$iteration.

We now give an asymptotic estimate for (4.5). Provided that $\lambda_{1}^{+} \leq \rho\left(x_{+}\right)<\lambda_{2}^{+}$and (4.4) holds, asymptotically, as $\rho\left(x_{+}\right) \rightarrow \lambda_{1}^{+}$, we have $\left(\lambda_{2}^{+}-\rho\left(x_{+}^{\prime}\right)\right) /\left(\lambda_{2}^{+}-\rho\left(x_{+}\right)\right) \rightarrow 1$ and therefore

$$
\begin{aligned}
& \frac{\rho\left(x_{+}^{\prime}\right)-\lambda_{1}^{+}}{\rho\left(x_{+}\right)-\lambda_{1}^{+}} \lesssim \sigma_{+}^{2} \\
& \text { with } \quad \sigma_{+}:=\sigma_{1,+}=\frac{\kappa_{+}+\gamma\left(2-\kappa_{+}\right)}{\left(2-\kappa_{+}\right)+\gamma \kappa_{+}} \quad \text { and } \quad \kappa_{+}:=\kappa_{1,+}=\frac{\left(\lambda_{1}^{+}-\lambda_{0}\right)\left(\lambda_{1}^{-}-\lambda_{2}^{+}\right)}{\left(\lambda_{2}^{+}-\lambda_{0}\right)\left(\lambda_{1}^{-}-\lambda_{1}^{+}\right)}
\end{aligned}
$$

Accordingly, our $\mathrm{PSD}^{+}$iteration converges at least linearly with the asymptotic convergence factor $\sigma_{+}^{2}$ that depends on the gap between $\lambda_{1}^{+}$and $\lambda_{0}$ relative to the gap between $\lambda_{2}^{+}$and $\lambda_{0}$, and of course on $\gamma$ : the quality measure of the preconditioner $T$. If $\lambda_{2}^{+} \approx \lambda_{1}^{+}$, then $\kappa_{+} \approx 1$ leads to a slower convergence of the $\mathrm{PSD}^{+}$iteration. Asymptotically, when $\lambda_{0} \rightarrow \lambda_{1}^{+}$, then $\kappa_{+} \rightarrow 0$ and therefore $\sigma_{+} \rightarrow \gamma$. Similar conclusions hold for (4.6). 
Corresponding convergence theorems for the block iterations $\mathrm{BPSD}^{+}$and $\mathrm{BPSA}^{-}$can be derived from [48]. For practically important (indefinite) LO(B)PCG type methods there are still no sharp convergence estimates. Convergence theorems proven for the preconditioned inverse iteration (PINVIT) [36], PSD/A [46, 47, 48], and our convergence theorems for the indefinite case can serve only as upper (non-sharp) estimates. Namely, the eigenvalue interlacing property implies that the Ritz value $\theta_{1}^{+}\left(\theta_{1}^{-}\right)$computed in Algorithm 3.1 with $m=3$ and $k_{+}=1$, $k_{-}=0\left(k_{+}=0, k_{-}=1\right)$ is at least as close to $\lambda_{1}^{+}\left(\lambda_{1}^{-}\right)$as the Ritz value $\rho\left(x_{+}^{\prime}\right)\left(\rho\left(x_{-}^{\prime}\right)\right)$ computed by $\mathrm{PSD}^{+}\left(\mathrm{PSA}^{-}\right)$since the subspace of $\mathrm{PSD}^{+}$and/or PSA ${ }^{-}$is contained in the subspace of Algorithm 3.1 with $m=3$. Thus, Algorithm 3.1 with $m=3$ and $k_{+}=1, k_{-}=0$ $\left(k_{+}=0, k_{-}=1\right)$ converges at least linearly with the asymptotic convergence factor $\sigma_{+}^{2}\left(\sigma_{-}^{2}\right)$.

5. Arbitrary spectral gaps. Algorithm 3.1 (and its extension derived at the end of Section 3) simultaneously computes a few eigenvalues around the definiteness interval and the corresponding eigenvectors of a given positive definite matrix pair. However, the definiteness interval is just one special spectral gap. In this section, we derive some ideas [43, Section 3.4] how to use the trace-reducing Algorithm 3.1 to compute a few eigenvalues around any spectral gap and the corresponding eigenvectors of a definite pair $(A, B)$.

Let $\lambda_{\min }$ denote the smallest and $\lambda_{\max }$ the largest finite eigenvalue of some positive definite matrix pair $(A, B)$ with finite eigenvalues (3.1) and let $\mathcal{I}_{0}$ denote its definiteness interval. ${ }^{5}$ For the given arbitrary shift $\lambda_{a} \in\left(\lambda_{\min }, \lambda_{\max }\right) \backslash \mathcal{I}_{0}$ that is not an eigenvalue of $(A, B)$, let $\mathcal{I}_{a}$ be the spectral gap around $\lambda_{a}$, that is, $\lambda_{a} \in \mathcal{I}_{a}$ and either $\mathcal{I}_{a}=\left(\lambda_{i}^{+}, \lambda_{i+1}^{+}\right)$, for some $i \in\left\{1, \ldots, n_{+}-1\right\}$ or $\mathcal{I}_{a}=\left(\lambda_{j+1}^{-}, \lambda_{j}^{-}\right)$, for some $j \in\left\{1, \ldots, n_{-}-1\right\}$. The spectral gap around $\lambda_{a}$ is defined analogously for a negative definite matrix pair. We want to simultaneously compute a small number of eigenvalues around the given shift $\lambda_{a}$, more precisely, the first $j_{b}$ eigenvalues that are bigger than $\lambda_{a}$ and the first $j_{s}$ eigenvalues that are smaller than $\lambda_{a}$, as well as the corresponding eigenvectors of a definite pair $(A, B)$. We transform the pair $(A, B)$ into some auxiliary positive definite matrix pair with the definiteness interval around zero and then use Algorithm 3.1 to compute $j_{b}+j_{s}$ eigenvalues around zero and the corresponding eigenvectors of that auxiliary pair, and consequently, the wanted eigenvalues around $\lambda_{a}$ and the corresponding eigenvectors of the pair $(A, B)$. We propose two ways to transform the given definite pair to some auxiliary one. ${ }^{6}$

Suppose first that $B$ is positive definite and $\lambda_{a}$ is from any desired spectral gap $\mathcal{I}_{a}$. Now the matrix pair $\left(A-\lambda_{a} B, B\right)$ has the desired spectral gap $\mathcal{I}:=\mathcal{I}_{a}-\lambda_{a}$ around zero and its eigenpair $(\mu, x)$ corresponds to the eigenpair $\left(\mu+\lambda_{a}, x\right)$ of the pair $(A, B)$. As is immediately verified, $\mathcal{I}$ is the definiteness interval of the positive definite pair

$$
\left(B^{-1},\left(A-\lambda_{a} B\right)^{-1}\right),
$$

which has the same eigenvalues as the pair $\left(A-\lambda_{a} B, B\right)$, the eigenvectors are just multiplied by $B$. Therefore, we can run Algorithm 3.1 with $\ell_{+}=j_{b}, \ell_{-}=j_{s}$ on the pair (5.1). Here, the preconditioned residual is obtained by a matrix vector product since $T=B$. This case can be extended to any definite pair with indefinite matrices and with the known definiteness interval, or, at least part of it. Let $\lambda_{0}$ be a definitizing shift of a positive definite pair $(A, B)$ with indefinite $A$ and $B$. Let $\lambda_{a}$ be from any desired spectral gap $\mathcal{I}_{a}$ of $(A, B)$. We assume that $\lambda_{0}$ and $\lambda_{a}$ are not relatively close. Then $A-\lambda_{0} B$ is positive definite and the eigenpair $(\lambda, x)$ of the pair $(A, B)$ corresponds to the eigenpair $\left(\lambda-\lambda_{0}, x\right)$ of the pair $\left(A-\lambda_{0} B, B\right)$. Now we can apply the previous case to the matrix pair $\left(B, A-\lambda_{0} B\right)$ in which

\footnotetext{
${ }^{5}$ If $B \preceq 0$, then $\mathcal{I}_{0}=\left(\lambda_{\max }, \infty\right)$. If $B \succeq 0$, then $\mathcal{I}_{0}=\left(-\infty, \lambda_{\min }\right)$.

${ }^{6}$ The author is indebted to Professor Krešimir Veselić for suggesting some of the ideas in the following, in particular for using (5.1) and providing Theorem 5.1 below.
} 
the eigenpair $\left(1 /\left(\lambda-\lambda_{0}\right), x\right)$ corresponds to the eigenpair $(\lambda, x)$ of the pair $(A, B)$. First, move the spectrum of $\left(B, A-\lambda_{0} B\right)$ to the left by $1 /\left(\lambda_{a}-\lambda_{0}\right)$, and then use the inverses. Therefore, $\mathcal{I}:=1 /\left(\mathcal{I}_{a}-\lambda_{0}\right)-1 /\left(\lambda_{a}-\lambda_{0}\right)$ is the definiteness interval around zero of the positive definite pair

$$
\left(\left(A-\lambda_{0} B\right)^{-1},\left(B-\left(\lambda_{a}-\lambda_{0}\right)^{-1}\left(A-\lambda_{0} B\right)\right)^{-1}\right),
$$

in which the eigenpair $\left(\frac{1}{\lambda-\lambda_{0}}-\frac{1}{\lambda_{a}-\lambda_{0}},\left(A-\lambda_{0} B\right) x\right)$ corresponds to the eigenpair $(\lambda, x)$ of the pair $(A, B)$. Here, the preconditioned residual is obtained by a matrix vector product since $T=A-\lambda_{0} B$. After running Algorithm 3.1 with $\ell_{+}=j_{s}, \ell_{-}=j_{b}$ (notice the change of the roles of $j_{s}$ and $j_{b}$ with this spectral transformation) on the pair (5.2), the computed eigenpair $(\mu, y)$ of the pair (5.2) corresponds to the eigenpair

$$
\left(\frac{1}{\mu+\left(\lambda_{a}-\lambda_{0}\right)^{-1}}+\lambda_{0},\left(A-\lambda_{0} B\right)^{-1} y\right)
$$

of the positive definite matrix pair $(A, B)$ with indefinite $A$ and $B$. A shortcoming of this way is that we must know the inverses or at least be able to easily solve linear systems of the type

$$
B x=c \quad \text { and } \quad\left(A-\lambda_{a} B\right) x=c
$$

for matrices in (5.1); and similarly for (5.2). There is another way, i.e., by using corresponding decompositions of the matrices appearing in (5.1) and (5.2). We assume first for simplicity $B=I$. Now make the indefinite decomposition ${ }^{7}$

$$
A-\lambda_{a} I=G J G^{H},
$$

where $\lambda_{a}$ is taken from any spectral gap $\mathcal{I}_{a}$ of $A$ and $J$ is Hermitian nonsingular, that is,

$$
J^{H}=J^{-1}=J
$$

and $G$ is a nonsingular lower block-triangular matrix with diagonal blocks of order 1 or 2 . If $J=I$ or $J=-I$, then $\lambda_{a}<\lambda_{\min }$ and $\lambda_{a}>\lambda_{\max }$, respectively, that is, $\lambda_{a}$ is not from any spectral gap. In this case, we can proceed, and at the end, compute the extremal eigenvalues, i.e., $\lambda_{\min }$ or $\lambda_{\max }$ and the ones closest to them. However, we are really interested in an indefinite $J=\operatorname{diag}( \pm 1)$.

Consider the auxiliary matrix pair $\left(G^{H} G, J\right)$. Due to (5.4), this pair has the same eigenvalues as the matrix $A-\lambda_{a} I$ and it possesses a set of $J$-orthonormal eigenvectors. More precisely, we have the following theorem; cf. [57, Section 3].

THEOREM 5.1. Let $A \in \mathbb{C}^{n \times n}$ be the given Hermitian matrix and let $U \in \mathbb{C}^{n \times p}, p \leq n$, have orthonormal columns spanning a spectral subspace of $A_{a}:=A-\lambda_{a} I$, that is,

$$
A_{a} U=U \Lambda_{a},
$$

\footnotetext{
${ }^{7}$ An indefinite decomposition of a Hermitian matrix $H$ is a decomposition of the form $P H P^{H}=L D L^{H}$, where $L$ is a unit lower triangular matrix, $D=D^{H}$ is a block-diagonal matrix with diagonal blocks of order 1 or 2 , and $P$ is a permutation matrix. This decomposition is obtained by the variants of the Bunch-Parlett decomposition $[1,10,11,12,13,15]$. An additional diagonalization of the diagonal blocks in $D$ and an appropriate scaling of the columns of $L$ results in a new decomposition $P H P^{H}=G J G^{H}$, where $G$ is a nonsingular lower block-triangular matrix with diagonal blocks of order 1 or 2 , and $J$ is a diagonal matrix of signs of the eigenvalues of $D$ (or, equivalently, of $H$ ) on its diagonal. We must pay attention to which pivoting strategy to use when performing an indefinite decomposition; for a banded matrix a pivoting strategy needs to preserve the bandwidth during the process $[12,14,28]$.
} 
where $\Lambda_{a}:=\Lambda-\lambda_{a} I_{p}, \Lambda \in \mathbb{R}^{p \times p}$ is a diagonal matrix containing some of the eigenvalues of A. Set

$$
F=G^{-1} U\left|\Lambda_{a}\right|^{1 / 2} \text { or, equivalently, } U=G F\left|\Lambda_{a}\right|^{-1 / 2}
$$

with $G, J$ from (5.3). Then

$$
F^{H} J F=\mathcal{J}
$$

with $\mathcal{J}:=\operatorname{sign}\left(\Lambda_{a}\right)$ and

$$
G^{H} G F=J F \Lambda_{a},
$$

that is, $F$ spans the corresponding spectral subspace of the pair $\left(G^{H} G, J\right)$. Conversely, (5.7) and (5.6) imply (5.5) and the fact that $U$ has orthonormal columns.

Proof. Premultiply $A_{a} U=U \Lambda_{a}$ by $A_{a}^{-1}$ and postmultiply by $\Lambda_{a}^{-1}$ to obtain

$$
U \Lambda_{a}^{-1}=A_{a}^{-1} U \text {. }
$$

Now,

$$
\begin{aligned}
F^{H} J F & =\left|\Lambda_{a}\right|^{1 / 2} U^{H} G^{-H} J G^{-1} U\left|\Lambda_{a}\right|^{1 / 2} \\
& =\left|\Lambda_{a}\right|^{1 / 2} U^{H} A_{a}^{-1} U\left|\Lambda_{a}\right|^{1 / 2} \\
& =\left|\Lambda_{a}\right|^{1 / 2} U^{H} U \Lambda_{a}^{-1}\left|\Lambda_{a}\right|^{1 / 2} \\
& =\left|\Lambda_{a}\right|^{1 / 2} \Lambda_{a}^{-1}\left|\Lambda_{a}\right|^{1 / 2}=\mathcal{J}
\end{aligned}
$$

with $\mathcal{J}=\operatorname{sign}\left(\Lambda_{a}\right)$. Equation (5.9) follows from (5.8). Further, premultiply (5.3) by $J G^{-1}$ to obtain

$$
G^{H}=J G^{-1} A_{a} .
$$

Now,

$$
\begin{aligned}
G^{H} G F & =G^{H} G G^{-1} U\left|\Lambda_{a}\right|^{1 / 2}=G^{H} U\left|\Lambda_{a}\right|^{1 / 2} \\
& =J G^{-1} A_{a} U\left|\Lambda_{a}\right|^{1 / 2}=J G^{-1} U \Lambda_{a}\left|\Lambda_{a}\right|^{1 / 2} \\
& =J G^{-1} U\left|\Lambda_{a}\right|^{1 / 2}\left|\Lambda_{a}\right|^{-1 / 2} \Lambda_{a}\left|\Lambda_{a}\right|^{1 / 2} \\
& =J F \Lambda_{a} .
\end{aligned}
$$

Equation (5.11) follows from (5.10) and (5.5).

Conversely, using (5.3), (5.7) and $J^{2}=I$ we have

$$
\begin{aligned}
A_{a} U & =G J G^{H} G F\left|\Lambda_{a}\right|^{-1 / 2} \\
& =G J J F \Lambda_{a}\left|\Lambda_{a}\right|^{-1 / 2} \\
& =G F\left|\Lambda_{a}\right|^{-1 / 2}\left|\Lambda_{a}\right|^{1 / 2} \Lambda_{a}\left|\Lambda_{a}\right|^{-1 / 2} \\
& =U \Lambda_{a} .
\end{aligned}
$$

Now,

$$
\begin{aligned}
U^{H} U & =\left|\Lambda_{a}\right|^{-1 / 2} F^{H} G^{H} G F\left|\Lambda_{a}\right|^{-1 / 2} \\
& =\left|\Lambda_{a}\right|^{-1 / 2} F^{H} J F \Lambda_{a}\left|\Lambda_{a}\right|^{-1 / 2} \\
& =\left|\Lambda_{a}\right|^{-1 / 2} \operatorname{sign}\left(\Lambda_{a}\right) \Lambda_{a}\left|\Lambda_{a}\right|^{-1 / 2}=I_{p} .
\end{aligned}
$$

Equation (5.12) follows from (5.7) and (5.13) follows from (5.6). 
Moreover, $\mathcal{I}:=\mathcal{I}_{a}-\lambda_{a}$ is the definiteness interval around zero of the positive definite pair $\left(G^{H} G, J\right)$. After running Algorithm 3.1 with $\ell_{+}=j_{b}, \ell_{-}=j_{s}$ on the pair $\left(G^{H} G, J\right)$, we get the diagonal matrix $\Lambda_{a}$ of the wanted Ritz values and the matrix $F$ of the corresponding Ritz vectors. Then the diagonal matrix $\Lambda=\Lambda_{a}+\lambda_{a} I_{p}$ contains the wanted eigenvalues around $\lambda_{a}$ and $U=G F\left|\Lambda_{a}\right|^{-1 / 2}$ contains the corresponding eigenvectors of the Hermitian matrix $A$. Instead of multiplying $G^{H}$ by $G$, we modify Algorithm 3.1 to work with factor $G$ in a way that $G^{H} G x$ is implemented by $G^{H}(G x)$ and a preconditioned residual $w=\operatorname{Tr}$ with $T=\left(G^{H} G\right)^{-1}$ is implemented by solving two linear systems $G^{H} z=r$ and $G w=z$. The first matrix $\left(U^{(i)}\right)^{H} G^{H} G U^{(i)}$ in a projected pair can be implemented by $\left(S^{(i)}\right)^{H} S^{(i)}$, where $S^{(i)}:=G U^{(i)}$.

If we have a pair $(A, B)$ with $B$ positive definite, then by making the Cholesky decomposition $B=L L^{H}$ the matrix $G$ in the preceding theorem has to be replaced by $L^{-1} G$. More precisely, we have the following proposition.

Proposition 5.2. Let $(A, B)$ be the given Hermitian matrix pair with $B \in \mathbb{C}^{n \times n}$ positive definite. Consider the indefinite decomposition

$$
A-\lambda_{a} B=C J C^{H},
$$

where $\lambda_{a}$ is taken from any spectral gap $\mathcal{I}_{a}$ of the pair $(A, B) ; J$ is as in (5.4) and the Cholesky decomposition $B=L L^{H}$. Let $V \in \mathbb{C}^{n \times p}, p \leq n$, be B-orthonormal: $V^{H} B V=I_{p}$, spanning a spectral subspace of the pair $\left(A-\lambda_{a} B, B\right)$, that is,

$$
\left(A-\lambda_{a} B\right) V=B V \Lambda_{a},
$$

where

$$
\Lambda_{a}:=\Lambda-\lambda_{a} I_{p},
$$

$\Lambda$ is a diagonal matrix containing some of the eigenvalues of the pair $(A, B)$. Set

$$
F=C^{-1} L L^{H} V\left|\Lambda_{a}\right|^{1 / 2} \text { or, equivalently, } V=L^{-H} L^{-1} C F\left|\Lambda_{a}\right|^{-1 / 2} .
$$

Then

$$
F^{H} J F=\mathcal{J}
$$

with $\mathcal{J}:=\operatorname{sign}\left(\Lambda_{a}\right)$ and

$$
\left(L^{-1} C\right)^{H}\left(L^{-1} C\right) F=J F \Lambda_{a},
$$

that is, $F$ spans the corresponding spectral subspace of the pair $\left(\left(L^{-1} C\right)^{H}\left(L^{-1} C\right), J\right)$. Conversely, (5.18) and (5.17) imply (5.15) and the fact that $V$ is B-orthonormal.

Proof. Set $A_{1}=L^{-1} A L^{-H}, U=L^{H} V$ and $G=L^{-1} C$. Premultiply (5.14) by $L^{-1}$ and postmultiply by $L^{-H}$, use $G=L^{-1} C$ to obtain

$$
A_{1}-\lambda_{a} I=G J G^{H} .
$$

Premultiply (5.15) by $L^{-1}$ and use $U=L^{H} V$ to obtain

$$
\left(A_{1}-\lambda_{a} I\right) U=U \Lambda_{a} .
$$

Now, $U^{H} U=V^{H} L^{H} L V=V^{H} B V=I_{p}$, set $F=G^{-1} U\left|\Lambda_{a}\right|^{1 / 2}$ and apply Theorem 5.1 with $\Lambda_{a}$ from (5.16) and substituting $A \rightarrow A_{1}$. 
Moreover, $\mathcal{I}:=\mathcal{I}_{a}-\lambda_{a}$ is the definiteness interval around zero of the positive definite pair $\left(\left(L^{-1} C\right)^{H}\left(L^{-1} C\right), J\right)$. After running Algorithm 3.1 with $\ell_{+}=j_{b}, \ell_{-}=j_{s}$ on this pair, we get the diagonal matrix $\Lambda_{a}$ of the wanted Ritz values and the matrix $F$ of the corresponding Ritz vectors. Then the diagonal matrix $\Lambda=\Lambda_{a}+\lambda_{a} I_{p}$ contains the wanted eigenvalues around $\lambda_{a}$ and $V=L^{-H} L^{-1} C F\left|\Lambda_{a}\right|^{-1 / 2}$ contains the corresponding eigenvectors of the matrix pair $(A, B)$ with $B$ positive definite. Here we modify Algorithm 3.1 to work with factors $L$ and $C$ in a way that $t=\left(L^{-1} C\right)^{H}\left(L^{-1} C\right) x$ is implemented by

$$
\begin{array}{ll}
y=C x & \text { matrix-vector multiplication, } \\
L z=y & \text { solving a linear system, } \\
L^{H} v=z & \text { solving a linear system, } \\
C^{H} v=t & \text { matrix-vector multiplication; }
\end{array}
$$

and similarly for preconditioned residuals with the preconditioner $T=\left(\left(L^{-1} C\right)^{H}\left(L^{-1} C\right)\right)^{-1}$.

REMARK 5.3. If we have a matrix pair $(A, B)$ with $B$ negative definite, then an analogous proposition holds; consider the auxiliary pair $\left(A-\lambda_{a} B,-B\right)$.

Finally, we give the proposition for a definite matrix pair $(A, B)$ with indefinite $B$ and $\lambda_{a}$ from any spectral gap.

Proposition 5.4. Let $(A, B)$ be the given positive definite matrix pair with $B \in \mathbb{C}^{n \times n}$ indefinite. Let $\lambda_{0}$ be an arbitrary definitizing shift and set $\widetilde{A}=A-\lambda_{0} B$, which is positive definite. Consider the indefinite decomposition $B-\left(\lambda_{a}-\lambda_{0}\right)^{-1} \widetilde{A}=C J C^{H}$, where $\lambda_{a}$ is taken from any spectral gap $\mathcal{I}_{a}$, of the pair $(A, B) ; J$ is as in (5.4) and the Cholesky

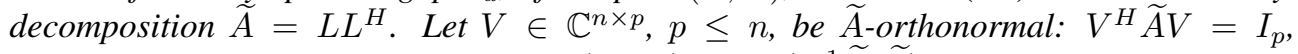
spanning a spectral subspace of the pair $\left(B-\left(\lambda_{a}-\lambda_{0}\right)^{-1} \widetilde{A}, \widetilde{A}\right)$, that is,

$$
\left(B-\left(\lambda_{a}-\lambda_{0}\right)^{-1} \widetilde{A}\right) V=\widetilde{A} V \Lambda_{0 a},
$$

where

$$
\Lambda_{0 a}:=\left(\Lambda-\lambda_{0} I_{p}\right)^{-1}-\left(\lambda_{a}-\lambda_{0}\right)^{-1} I_{p}
$$

$\Lambda \in \mathbb{R}^{p \times p}$ is a diagonal matrix containing some of the eigenvalues of the pair $(A, B)$. Set

$$
F=C^{-1} L L^{H} V\left|\Lambda_{0 a}\right|^{1 / 2} \text { or, equivalently, } V=L^{-H} L^{-1} C F\left|\Lambda_{0 a}\right|^{-1 / 2} .
$$

Then

$$
F^{H} J F=\mathcal{J}
$$

with $\mathcal{J}:=\operatorname{sign}\left(\Lambda_{0 a}\right)$ and

$$
\left(L^{-1} C\right)^{H}\left(L^{-1} C\right) F=J F \Lambda_{0 a}
$$

that is, $F$ spans the corresponding spectral subspace of the pair $\left(\left(L^{-1} C\right)^{H}\left(L^{-1} C\right), J\right)$. Conversely, (5.21) and (5.20) imply (5.19) and the fact that $V$ is $\widetilde{A}$-orthonormal.

Proof. The proof follows immediately from Theorem 5.2 by substituting $A-\lambda_{a} B$ with $B-\left(\lambda_{a}-\lambda_{0}\right)^{-1} \widetilde{A}, B$ with $\widetilde{A}$ and $\Lambda_{a}$ with $\Lambda_{0 a}$.

Moreover, $\mathcal{I}:=1 /\left(\mathcal{I}_{a}-\lambda_{0}\right)-1 /\left(\lambda_{a}-\lambda_{0}\right)$ is the definiteness interval around zero of the positive definite pair $\left(\left(L^{-1} C\right)^{H}\left(L^{-1} C\right), J\right)$, and we can use Algorithm 3.1 with $\ell_{+}=j_{s}, \ell_{-}=j_{b}$; notice the change of the indices \pm compared to the previous cases. After running Algorithm 3.1, $\Lambda=\left(\Lambda_{0 a}+\left(\lambda_{a}-\lambda_{0}\right)^{-1} I_{p}\right)^{-1}+\lambda_{0} I_{p}$ contains the wanted eigenvalues around $\lambda_{a}$ and $V=L^{-H} L^{-1} C F\left|\Lambda_{0 a}\right|^{-1 / 2}$ contains the corresponding eigenvectors of 
the positive definite matrix pair $(A, B)$ with indefinite $B$. Although $V^{H} \widetilde{A} V=I_{p}$, after premultiplication (5.19) by $V^{H}$ we get $V^{H} B V=\Lambda_{0 a}+\left(\lambda_{a}-\lambda_{0}\right)^{-1} I_{p}$. Therefore, $V$ is $B$-orthogonal and after normalization it becomes $B$-orthonormal.

REMARK 5.5. If a matrix pair $(A, B)$ is negative definite with $B$ indefinite, then an analogous proposition holds; consider the auxiliary pair $\left(B-\left(\lambda_{a}-\lambda_{0}\right)^{-1} \widetilde{A},-\widetilde{A}\right)$.

REMARK 5.6. When applying Algorithm 3.1 to the corresponding pair from (5.7) or (5.21), an initial guess $X^{(0)}$ needs to have at least $k_{+} J$-positive columns and $k_{-} J$ negative columns. This is easily achieved by choosing appropriate columns of the identity matrix. However, when using inverses, the initial guess needs to have at least $k_{+} \widetilde{B}^{-1}$-positive columns and $k_{-} \widetilde{B}^{-1}$-negative columns. For the corresponding pair (5.1), $\widetilde{B}=A-\lambda_{a} B$, and for (5.2), $\widetilde{B}=B-\left(\lambda_{a}-\lambda_{0}\right)^{-1}\left(A-\lambda_{0} B\right)$. Assume $Y^{H} J Y=J_{k}$ for some matrix $Y \in \mathbb{C}^{n \times k}$, where $J_{k}=\operatorname{diag}( \pm 1)$ and $k=k_{+}+k_{-}$. Then for $X:=C Y$ we have $X^{H} \widetilde{B}^{-1} X=J_{k}$, where $\widetilde{B}=C J C^{H}$. Therefore, $C X^{(0)}$ can be used as an initial guess for our algorithm applied to the corresponding pair (5.1) or (5.2).

6. Numerical experiments. In this section, we consider some numerical experiments illustrating the performance of Algorithm 3.1 for different $k_{ \pm}=\ell_{ \pm}$, the number of wanted eigenpairs, and different $m$; the dimension of the search subspace is then $\left(k_{+}+k_{-}\right) m$. In some experiments we compare different preconditioners for the same initial guess, $k_{ \pm}$, and $m$. In all experiments we use tol $=10^{-7}$ in the convergence criterion (3.6); unless otherwise stated. Sometimes our algorithm fails to converge within the allowed number of iterations. Such failure is marked with $\infty$ in the results. We have two sets of experiments. In the first set we apply Algorithm 3.1 to the original matrix pair to compute eigenpairs around the definiteness interval. In the second set we apply Algorithm 3.1 to the transformed matrix pair to compute eigenpairs around some arbitrary spectral gap. All experiments ${ }^{8}$ have been performed in MATLAB R2014a on Intel Core i3-4150 CPU 3.50GHz, 6 GB RAM, with the exception of Example 6.2, which has been performed in MATLAB R2014a on Intel i5 760 @ $2.80 \mathrm{GHz}, 8 \mathrm{~GB}$ RAM.

6.1. The definiteness interval. In this section, we consider one product eigenvalue problem and two hyperbolic quadratic eigenvalue problems. We apply Algorithm 3.1 to the corresponding definite matrix pair. A comparison is made between Algorithm 3.1 for several values of $m$ and the corresponding algorithm that uses all previous iteration matrices $X^{(j)}$, that is, $P^{(j)}$ for all $j \leq i$ denoted by "w.h." in the results; meaning the whole history.

EXAMPLE 6.1. Consider the product eigenvalue problem

$$
M K x=\lambda^{2} x, \quad K M y=\lambda^{2} y, \quad 0 \neq x, y \in \mathbb{C}^{n},
$$

where $M, K \in \mathbb{C}^{n \times n}$ are Hermitian positive semidefinite and one of them is positive definite. This problem appears in computational quantum chemistry [3], where it is of interest to find a few smallest eigenvalues and the corresponding eigenvectors. Bai and Li developed the corresponding theory (Cauchy-like interlacing inequalities, the trace minimization principle) for this problem in [3]. The BPSD-like method for the product eigenvalue problem is proposed in [53] and LOBPCG-like methods in $[4,5,6]$. It is well known that (6.1) is equivalent to the GEP for the pair $(A, B)$, where

$$
A=\left[\begin{array}{cc}
K & 0 \\
0 & M
\end{array}\right], \quad B=\left[\begin{array}{cc}
0 & I \\
I & 0
\end{array}\right] .
$$

\footnotetext{
${ }^{8}$ The codes are available on the personal website of the author with the url: https://www. mathos . unios. hr/index.php/kadrovi/nastavnici-i-suradnici/130 under Software.
} 


\section{ETNA}

Kent State University and

Johann Radon Institute (RICAM)

Therefore, $\lambda^{2}$ is an eigenvalue of $M K$ if and only if $\pm \lambda$ are the eigenvalues of $(A, B)$, the corresponding eigenvectors of $(A, B)$ are given by

$$
\left[\begin{array}{l}
x \\
y
\end{array}\right], \quad\left[\begin{array}{c}
x \\
-y
\end{array}\right],
$$

where $x, y$ are from (6.1). If both $K$ and $M$ are positive definite, then the pair $(A, B)$ from (6.2) is trivially positive definite, and finding the smallest eigenvalues of the product eigenvalue problem (6.1) is equivalent to finding the eigenvalues around the definiteness interval of the pair $(A, B)$. The direct application of our Algorithm 3.1 to the pair $(A, B)$ would imply working with matrices of double order. Therefore, a specialized LOBPCGtype algorithm has been proposed in [38, Algorithm 3]. This algorithm is mathematically equivalent to [4, Algorithm 4.1] and the experiment [38, Example 5.1] demonstrates that these two algorithms have similar convergence behavior. Here we want to compare the behavior of the specialized Algorithm 3.1 for different values of $m$. This specialized algorithm reduces memory requirements, computational cost, and CPU time, and preserves the symmetry of the computed eigenpairs, compared with the direct application of Algorithm 3.1 to the product eigenvalue problem. For brevity, we will not present this algorithm, but we refer the reader to [38, Algorithm 3], where this specialized algorithm is shown for $m=3$. As a concrete example we pick the linear response analysis of the density matrix in electronic structure calculations using the same matrices as in [6], [38, Example 5.1]. Thus, the matrices $K, M$ are real, symmetric positive definite, and of order 5660. We refer to [6, Section 5] for more information about these matrices and the natural preconditioner for this concrete example.

TABLE 6.1

Product eigenvalue problem from Example 6.1 with a zero shift using exact and CG preconditioners.

\begin{tabular}{|c|c|c|c|c|c|c|}
\hline & $m$ & 2 & 3 & 4 & 5 & w.h. \\
\hline \multicolumn{7}{|c|}{$k_{+}=1$} \\
\hline exact & $\#$ iter & 107 & 41 & 35 & 35 & 35 \\
\hline \multirow{3}{*}{ CG } & \# iter & 108 & 41 & 38 & 37 & 35 \\
\hline & \# in.iter & 725,737 & 355,353 & 327,325 & 319,319 & 302,298 \\
\hline & av.\# in.iter & $6.78,6.89$ & $8.88,8.23$ & $8.84,8.78$ & $8.86,8.86$ & $8.88,8.76$ \\
\hline & \multicolumn{6}{|c|}{$k_{+}=2$} \\
\hline exact & $\#$ iter & 97,116 & 38,47 & 34,39 & 34,34 & 33,33 \\
\hline \multirow{3}{*}{ CG } & $\#$ iter & 98,119 & 38,48 & 35,40 & 34,34 & 32,33 \\
\hline & \# in.iter & 1411,1440 & 741,728 & 642,625 & 578,561 & 550,538 \\
\hline & av.\# in.iter & $6.56,6.7$ & $8.82,8.67$ & $8.79,8.56$ & $8.76,8.50$ & $8.73,8.54$ \\
\hline & \multicolumn{6}{|c|}{$k_{+}=3$} \\
\hline exact & $\#$ iter & $96,103,107$ & $37,38,47$ & $34,36,40$ & $33,34,36$ & $30,32,33$ \\
\hline \multirow{3}{*}{ CG } & $\#$ iter & $96,103,120$ & $37,39,49$ & $35,36,42$ & $34,35,37$ & $29,31,34$ \\
\hline & \# in.iter & 2042,1990 & 1063,1039 & 972,959 & 912,904 & 794,782 \\
\hline & av.\# in.iter & $6.46,6.3$ & $8.71,8.52$ & $8.84,8.72$ & $8.85,8.78$ & $8.73,8.59$ \\
\hline & \multicolumn{6}{|c|}{$k_{+}=4$} \\
\hline exact & $\#$ iter & $95,96,104,227$ & $36,37,39,70$ & $33,34,35,69$ & $32,33,33,67$ & $29,29,30,36$ \\
\hline \multirow{3}{*}{ CG } & $\#$ iter & $98,98,102,222$ & $35,38,40,67$ & $34,35,35,71$ & $33,33,34,65$ & $29,30,30,36$ \\
\hline & \# in.iter & 3355,3306 & 1549,1506 & 1518,1479 & 1424,1406 & 1029,1018 \\
\hline & av.\# in.iter & $6.50,6.41$ & $8.80,8.56$ & $8.88,8.65$ & $8.84,8.73$ & $8.50,8.41$ \\
\hline
\end{tabular}


The four smallest positive eigenvalues are given by

$$
\begin{array}{ll}
\lambda_{1}^{+} \approx 0.541812517132466, & \lambda_{2}^{+} \approx 0.541812517132473, \\
\lambda_{3}^{+} \approx 0.541812517132498, & \lambda_{4}^{+} \approx 0.615143209274579 .
\end{array}
$$

A comparison is made between the specialized Algorithm 3.1 with $m=2,3,4,5$, and the corresponding algorithm in which the whole history is included. Results are reported in Table 6.1. The rows in this table correspond to one value of $k_{+}$for $k_{+}=1,2,3,4$, and the columns correspond to one value of $m$. Here we use zero as a definitizing shift and two different ways for computing preconditioned residuals, i.e., the MATLAB backs lash operator, and the linear conjugate gradient method with stopping tolerance $10^{-2}$ and a maximum of 20 iterations; the rows in the table starting with "exact" and "CG," respectively. Since we use a zero shift, the preconditioner $T$ equals $A^{-1}$ for $A$ from (6.2). Hence, we apply the CG method twice: once for $K$ and once for $M$. The total number of CG iterations is denoted by "\# in.iter" in Table 6.1; the first number corresponds to the total number for $K$ and the second for $M$. We also put the average number of CG iterations per iteration of the specialized Algorithm 3.1 denoted by "av.\# in.iter," first for $K$ and then for $M$. The total number of required iterations of the specialized Algorithm 3.1 until all $k_{+}$desired eigenvalues have converged is denoted by "\# iter." For example, we explain the field by $m=2$ and $k_{+}=3$ from Table 6.1: All three wanted eigenvalues converge after 107 iterations of Algorithm 3.1 when using the exact preconditioner, and after 120 iterations when using CG-based preconditioners. The first smallest positive eigenvalue converges after 96 iterations, and the second smallest positive converges after 103 iterations. The total number of inner CG iterations for $K$ and $M$ is 2042 and 1990, respectively. The average number of inner CG iterations per iteration of the specialized Algorithm 3.1 for $K$ and $M$ is 6.46 and 6.3 , respectively.

The findings of Table 6.1 are as follows. The total number of required iterations of the specialized Algorithm 3.1, and also the total number of inner CG iterations, is reduced by increasing the dimension of the search subspace. The most important difference is between $m=2$ and $m=3$; specialized indefinite BPSD/A and specialized indefinite LOBPCG, respectively. The total number of required iterations of the specialized variant of Algorithm 3.1 for $m=3,4,5$, and when using the whole history is very similar, the largest difference is for the fourth Ritz value. The first three positive eigenvalues are clustered, therefore the convergence for the first three Ritz pairs is much faster than for the fourth one. Moreover, by enlarging the block size $k_{+}$we can get a faster convergence to the smallest eigenpair, but with more numerical cost per iteration of the specialized Algorithm 3.1. It is very important to note that the results for CG-based preconditioners with very crude approximations of the preconditioned residuals are very similar, in some cases the same, to the results for exact preconditioned residuals. The maximum number of inner CG iterations per iteration of the specialized Algorithm 3.1 is 10 for all values of $k_{+}$and $m$ including the case in which the whole history is included. We have repeated the experiment with CG-based preconditioners with stopping tolerance $10^{-1}$ and $10^{-3}$; the total number of required iterations of the specialized Algorithm 3.1 is quite similar to the presented case.

Now we choose a relatively close definitizing shift $\lambda_{0}=0.54$ so we make use of the preconditioners $T_{ \pm}=\left(A \mp \lambda_{0} B\right)^{-1}$. We apply the linear CG method with stopping tolerance $10^{-2}$ and a maximum of 50 iterations. Table 6.2 gives the total number of required iterations of the specialized Algorithm 3.1 until all $k_{+}=1,2,3,4$ desired eigenpairs have converged, including the total and the average number of $\mathrm{CG}$ iterations for $m=2,3,4,5$, and when using the whole history. For example, the total number of inner CG iterations for $k_{+}=1$ is 158 for $m=2$ and in all other cases it is 161 . The average number of inner CG iterations per iteration of the specialized Algorithm 3.1 for $k_{+}=1$ is 31.6 for $m=2$ and it is 32.2 in all other cases. 
TABLE 6.2

Product eigenvalue problem from Example 6.1 with a nonzero shift using CG preconditioners.

\begin{tabular}{c|rr|rr|rr} 
& \multicolumn{2}{|c}{$k_{+}=1$} & \multicolumn{2}{c}{$k_{+}=2$} & \multicolumn{2}{c}{$k_{+}=3$} \\
\hline$m$ & 2 & $3,4,5$, w.h. & 2 & $3,4,5$, w.h. & 2 & $3,4,5$, w.h. \\
\hline \# iter & 6 & 6 & 6,6 & 6,6 & $6,6,6$ & $6,6,6$ \\
\# in.iter & 158 & 161 & 310 & 311 & 471 & 476 \\
av.\# in.iter & 31.6 & 32.2 & 31.0 & 31.1 & 31.4 & 31.73 \\
\hline & \multicolumn{7}{r}{$k_{+}=4$} \\
\hline$m$ & 2 & 3 & 4 & 5 & w.h. \\
\hline \# iter & $6,6,6,46$ & $6,6,6,18$ & $6,6,6,18$ & $6,6,6,18$ & $6,6,17$ \\
\# in.iter & 1,251 & 908 & 908 & 911 & 881 \\
av.\# in.iter & 20.85 & 28.38 & 28.38 & 28.47 & 28.42
\end{tabular}

The maximum number of inner CG iterations per iteration of the specialized Algorithm 3.1 is reached in some cases. When an excellent shift is used, there is no significant difference in the convergence of the fourth Ritz value by enlarging the dimension of the search subspace.

EXAMPLE 6.2. Consider a quadratic eigenvalue problem (QEP)

$$
\left(\lambda^{2} M+\lambda D+K\right) x=0, \quad 0 \neq x \in \mathbb{C}^{n},
$$

where $M, D, K \in \mathbb{C}^{n \times n}$ are Hermitian and $M$ is positive definite. A Hermitian linearization of (6.3) yields the Hermitian pair $(A, B)$ with

$$
A=\left[\begin{array}{cc}
M & 0 \\
0 & -K
\end{array}\right], \quad B=\left[\begin{array}{cc}
0 & M \\
M & D
\end{array}\right]
$$

The positive definiteness of this matrix pair is equivalent to the hyperbolicity of the original QEP [26, 57].

Here we consider a simple scalable example as in [38, Example 5.2]:

$$
K=(n+1)^{2}\left[\begin{array}{cccc}
2 & -1 & & \\
-1 & \ddots & \ddots & \\
& \ddots & \ddots & -1 \\
& & -1 & 2
\end{array}\right], \quad M=I_{n}, \quad D=2 K
$$

This QEP is hyperbolic; its eigenvalues are given by

$$
\lambda_{j}^{ \pm}=-\alpha_{j} \pm \sqrt{\alpha_{j}^{2}-\alpha_{j}}, \quad \text { where } \quad \alpha_{j}=4(n+1)^{2} \sin ^{2} \frac{j \pi}{2(n+1)},
$$

for $j=1, \ldots, n$. As $n$ increases, the definiteness interval of $(A, B)$ converges to around $(-19.2258,-0.5134)$. Considering that $\|D\|_{2}=2\|K\|_{2}=O\left(n^{2}\right)$, while $\|M\|_{2}=1$, we propose to rescale the pair $(A, B)$ as follows:

$$
(A, B) \leftarrow\left[\begin{array}{ll}
I & \\
& \frac{1}{n+1} I
\end{array}\right](A, B)\left[\begin{array}{ll}
I & \\
& \frac{1}{n+1} I
\end{array}\right] .
$$




\section{ETNA}

Kent State University and

Johann Radon Institute (RICAM)

TABLE 6.3

QEP from Example 6.2 with $A M G$ preconditioners.

\begin{tabular}{c|ccccr}
$m$ & 2 & 3 & 4 & 5 & w.h. \\
\hline$n$ & \multicolumn{5}{|c}{ one shift, without deflation } \\
\hline 2000 & $\infty(7.36)$ & $221(7.18)$ & $153(7.79)$ & $172(11.25)$ & $214(6020)$ \\
4000 & $\infty(12.73)$ & $\infty(18.55)$ & $245(25.65)$ & $192(23.23)$ & $16(4.06)$ \\
6000 & $\infty(19.09)$ & $240(21.32)$ & $206(29.81)$ & $132(22.17)$ & $17(7.91)$ \\
8000 & $\infty(24.78)$ & $234(26.77)$ & $196(36.72)$ & $63(12.89)$ & $12(3.57)$ \\
\hline$n$ & \multicolumn{5}{c}{ two shifts, without deflation } \\
\hline 2000 & $81(1.69)$ & $21(0.55)$ & $21(0.94)$ & $19(1.07)$ & $14(1.86)$ \\
4000 & $68(2.70)$ & $22(1.15)$ & $23(1.60)$ & $23(2.35)$ & $24(14.1)$ \\
6000 & $53(3.16)$ & $26(2.29)$ & $20(2.28)$ & $18(2.20)$ & $14(4.83)$ \\
8000 & $37(3.12)$ & $18(2.09)$ & $20(2.65)$ & $21(3.64)$ & $61(335)$ \\
\hline$n$ & \multicolumn{5}{|c}{ two shifts, with deflation } \\
\hline 2000 & $\infty(1.69)$ & $\infty(1.41)$ & $24(0.58)$ & $17(0.63)$ & $18(1.63)$ \\
4000 & $\infty(2.83)$ & $19(0.70)$ & $\infty(3.25)$ & $22(1.11)$ & $16(2.21)$ \\
6000 & $\infty(3.43)$ & $25(1.27)$ & $27(1.53)$ & $21(1.55)$ & $15(2.77)$ \\
8000 & $\infty(4.63)$ & $20(1.44)$ & $20(1.87)$ & $21(2.10)$ & $17(6.37)$
\end{tabular}

We aim at computing the eigenvalues $\lambda_{j}^{ \pm}$for $j=1,2,3$, that is, $k_{ \pm}=3$ in Algorithm 3.1 and the variant of Algorithm 3.1 with two preconditioners; see the end of Section 3. A comparison is made between Algorithm 3.1 with $m=2,3,4,5$, and the corresponding algorithm in which the whole history is included. The number of total iterations for $B$-positive and $B$-negative eigenpairs for $n=2000,4000,6000,8000$ is reported in Table 6.3. Notice that the order of the linearized pair $(A, B)$ is doubled, so $n=8000$ means that we work with matrices $A, B$ of order 16,000. The maximum number of allowed iterations of Algorithm 3.1 is 300 for one shift and 100 for two shifts. The CPU time is given in brackets in Table 6.3. An algebraic multigrid (AMG) V-cycle preconditioner can be used as a black box for solving linear systems for the preconditioned residuals in this example since we consider the scalable pair $(A, B)$. Therefore, we use the implementation HSL_MI20 [27] with the default settings. Since $D$ in (6.5) is positive definite, for fixed $n$ and $B$ given in (6.4), the initial $B$-positive and $B$-negative vectors are chosen as corresponding columns from $[0 ; I]$ and $\left[M^{-1} D ;-I\right]$, respectively. We use the same initial guess for fixed $n$ and fixed shifts.

The findings of Table 6.3 are as follows. Enlarging the dimension of the search subspace significantly reduces, in almost all cases, the number of total iterations of Algorithm 3.1 when the shift is not good (in this case, $\lambda_{0}=-5$ ), but when using two excellent shifts (here, $\lambda_{0}^{+}=-0.514$ and $\left.\lambda_{0}^{-}=-19.22\right)$, there is no such significant reduction in the number of total iterations in the indefinite $(m)$-scheme when $m$ is increased. Although in almost all cases the use of all previous iteration matrices gives the smallest number of total iterations, there is an increase in the numerical cost and memory requirements per iteration. When the dimension of the search subspace is fixed, like in Algorithm 3.1, there is a fixed numerical cost and memory requirements per iteration. When two excellent shifts are used, we see that enlarging the dimension of the search subspace increases CPU time. Again, the indefinite $(m)$-scheme with $m=3$, i.e., indefinite LOBPCG, is more efficient than the indefinite $(m)$-scheme with $m=2$, i.e., indefinite BPSD/A. We notice that the number of total iterations of Algorithm 3.1 with two shifts for fixed $m$ is very similar for different values of $n$. 
EXAMPLE 6.3. Consider another hyperbolic quadratic eigenvalue problem with matrices

$$
K=\left[\begin{array}{cccc}
15 & -5 & & \\
-5 & \ddots & \ddots & \\
& \ddots & \ddots & -5 \\
& & -5 & 15
\end{array}\right], \quad M=I_{n}, \quad D=2 K
$$

These matrices can be produced by the command nlevp ('spring', n, 1, 10, 5, 10, 5) from the NLEVP collection [8] and the eigenvalues are given by

$$
\lambda_{j}^{ \pm}=-\alpha_{j} \pm \sqrt{\alpha_{j}^{2}-\alpha_{j}}, \quad \text { where } \quad \alpha_{j}=5\left(3-2 \cos \frac{j \pi}{n+1}\right),
$$

for $j=1, \ldots, n$. As $n$ increases, the definiteness interval of the linearized pair $(A, B)$ from (6.4) converges to around $(-9.4721,-0.52786)$. Moreover, the gaps between eigenvalues become arbitrarily small as $n \rightarrow \infty$. The illustration for $n=1000$ is given in Fig. 6.1.
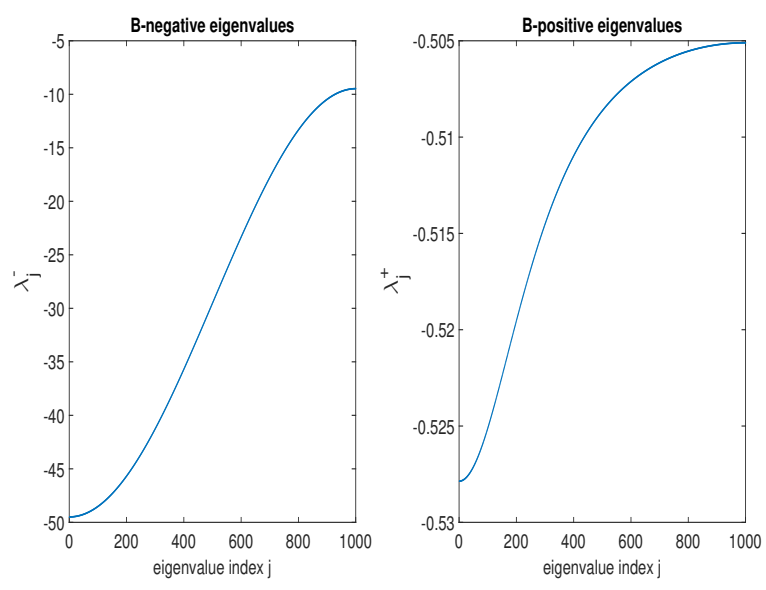

FIG. 6.1. Eigenvalues of Example 6.3 for $n=1000$. The definiteness interval of the pair $(A, B)$, when $n$ increases, is around $(-9.4721,-0.52786) .1000 B$-negative eigenvalues are in the interval $(-49.4948,-9.4721)$ and 1000 B-positive eigenvalues are in the interval $(-0.52786,-0.5051)$.

The inefficiency of Algorithm 3.1 with $m=3$ (since it uses only one preconditioner) is illustrated in [38, Example 5.3] caused by a decrease in the eigenvalue gaps; illustration was made using the exact preconditioner with shift $\lambda_{0}=-5$, nearly in the middle of the definiteness interval. However, exact tailored preconditioners $T_{ \pm}$perform very well. Here we want to compare Algorithm 3.1 with two shifts and with $m=2,3,4,5,10$, and when using the whole history. Here we use $\lambda_{0}^{-}=-9.47$ and $\lambda_{0}^{+}=-0.528$ as definitizing shifts and two different ways for computing preconditioned residuals: the MATLAB backslash operator and the linear CG method with stopping tolerance $10^{-2}$ and a maximum of 50 iterations. The obtained results for exact preconditioners and CG-based preconditioners are reported in Table 6.4 and Table 6.5, respectively. We aim at computing the eigenvalues $\lambda_{j}^{ \pm}$for $j=1,2,3$, that is, $k_{ \pm}=3$ in the variant of Algorithm 3.1 with two preconditioners. We list the number of required iterations for $B$-positive and $B$-negative eigenpairs to converge separately, denoted by "\# iter $B$-pos" and "\# iter $B$-neg", respectively, in Tables 6.4 and 6.5 for $n=1000$ and $n=2000$; note that the order of the pair $(A, B)$ is doubled. The CPU time is also given 
TABLE 6.4

QEP from Example 6.3 with two shifts and exact inverse preconditioners.

\begin{tabular}{c|rrrrrr}
$m$ & 2 & 3 & 4 & 5 & 10 & w.h. \\
\hline & \multicolumn{7}{|c}{$n=1000$} \\
\hline \# iter B-pos & 227 & 37 & 30 & 28 & 23 & 20 \\
\# iter B-neg & 19 & 10 & 10 & 9 & 9 & 9 \\
CPU time & $(1.74)$ & $(0.35)$ & $(0.36)$ & $(0.39)$ & $(0.48)$ & $(0.84)$ \\
\hline & 720 & 73 & 62 & 61 & 49 & 36 \\
\hline \# iter B-pos & 54 & 17 & 16 & 15 & 14 & 14 \\
\# iter B-neg & $(11.9)$ & $(1.30)$ & $(1.63)$ & $(1.76)$ & $(2.62)$ & $(7.35)$ \\
CPU time & & & & & &
\end{tabular}

TABLE 6.5

QEP from Example 6.3 with two shifts and CG-based preconditioners.

\begin{tabular}{c|rrrrrr}
$m$ & 2 & 3 & 4 & 5 & 10 & w.h. \\
\hline & \multicolumn{7}{|c}{$n=1000$} & & & \\
\hline \# iter B-pos & 239 & 51 & 47 & 42 & 37 & 35 \\
\# iter B-neg & 288 & 79 & 79 & 79 & 80 & 77 \\
\# in.iter B-pos & 23,942 & 6950 & 6400 & 6000 & 5300 & 5100 \\
\# in.iter B-neg & 34,350 & 11,550 & 11,500 & 11,500 & 11,600 & 11,350 \\
CPU time & $(8.06)$ & $(1.22)$ & $(1.54)$ & $(1.70)$ & $(2.55)$ & $(7.38)$ \\
\hline & 716 & 89 & 86 & 77 & 72 & 67 \\
\hline \# iter B-pos & 716 & 151 & 151 & 151 & 113 \\
\# iter B-neg & 941 & 151 & 11,800 & 11,200 & 10,500 & 9900 \\
\# in.iter B-pos & 71,696 & 12,750 & 11,500 \\
\# in.iter B-neg & 90,050 & 24,450 & 22,450 & 22,450 & 22,400 & 16,800 \\
CPU time & $(26.56)$ & $(5.93)$ & $(8.39)$ & $(8.70)$ & $(14.38)$ & $(271.9)$
\end{tabular}

in both tables. The total number of inner CG-iterations is given in Table 6.5, separately for preconditioned residuals corresponding to $B$-positive and $B$-negative Ritz vectors, denoted by "\# in.iter $B$-pos" and "\# in.iter $B$-neg", respectively.

The findings of Tables 6.4 and 6.5 are as follows. The total number of required iterations of Algorithm 3.1 with two shifts, and also the total number of inner CG iterations, are reduced by increasing the dimension of the search subspace. The most important difference is again between $m=2$ and $m=3$. Significant savings in CPU time are achieved by switching from $m=2$ to $m=3$. Algorithm 3.1 needs more CPU time by a further increase in the dimension of the search subspace, especially when using the whole history. The effectiveness of the tailored preconditioners $\left(A-\lambda_{0}^{ \pm} B\right)^{-1}$ deteriorates as $n$ increases, due to the decrease in the eigenvalue gaps. In this example, in contrast to Example 6.1, the exact preconditioners (note that matrices $A, B$ are sparse) have outperformed the CG-based preconditioners.

Finally, we demonstrate that using a shift $\lambda_{0}^{+}$that is very close to the eigenvalue $\lambda_{1}^{+}$ accelerates the convergence of Algorithm 3.1, as observed in Section 4.1. We list the required number of iterations for all six eigenpairs separately in Table 6.6.

6.2. The arbitrary gaps. In this section, we consider ordinary eigenvalue problems with indefinite and positive definite matrices, as well as a definite generalized eigenvalue problem with both matrices indefinite. A comparison is made between Algorithm 3.1 applied to the corresponding pair (5.1) or (5.2), and to the corresponding pair from (5.7) or (5.21). 


\section{ETNA}

Kent State University and

Johann Radon Institute (RICAM)

TABLE 6.6

QEP from Example 6.3 with two exact inverse preconditioners with shifts $\lambda_{0}^{-}=-9.47$ and $\lambda_{0}^{+}=-0.528$, $\left(\lambda_{0}^{+}=-0.5279\right)$ for $n=1000$.

\begin{tabular}{c|cccccc|c}
$m$ & $\lambda_{3}^{-}$ & $\lambda_{2}^{-}$ & $\lambda_{1}^{-}$ & $\lambda_{1}^{+}$ & $\lambda_{2}^{+}$ & $\lambda_{3}^{+}$ & CPU time \\
\hline 2 & 19 & 13 & 11 & $133(41)$ & $157(49)$ & $227(71)$ & $1.74(0.66)$ \\
3 & 10 & 9 & 8 & $35(19)$ & $36(21)$ & $37(24)$ & $0.35(0.27)$ \\
10 & 9 & 8 & 8 & $22(14)$ & $22(15)$ & $23(15)$ & $0.48(0.37)$
\end{tabular}

In our results, the former is denoted by "using (an) inverse(s)" and the latter by "using (a) decomposition(s)." An indefinite decomposition of a particular matrix is obtained by [54, pp. 1-2]. A maximum number of allowed iterations is 100 in all experiments. All our results are reported in tables, where for a fixed $k_{ \pm}$:

(i) the first column corresponds to the total number of iterations of Algorithm 3.1,

(ii) the second column corresponds to the 2-norm of the absolute error $A V-B V \Lambda$, where $\Lambda$ is a diagonal matrix of approximations of the wanted eigenvalues, and $V$ is a matrix of approximations of the corresponding eigenvectors,

(iii) the third column corresponds to CPU time; the execution time of Algorithm 3.1 applied to the transformed matrix pair.

Although the initial guesses when using inverses and decompositions are connected (see Remark 5.6), due to roundoff, we can expect a different total number of iterations of our algorithm when using inverses and decompositions. Notice that $T=I$, when applying our algorithm to the pair $\left(I,\left(A-\lambda_{a} I\right)^{-1}\right)$, while $T=\left(G^{H} G\right)^{-1}$, when applying our algorithm to the pair $\left(G^{H} G, J\right)$.

EXAMPLE 6.4. We first consider two sparse ordinary eigenvalue problems with real symmetric indefinite matrices originating from Platzman's oceanographic models. These problems are part of the Harwell-Boeing collection [18]. We obtain them from the Matrix Market [9]. All eigenvalues occur in pairs, with the exception of an isolated singleton at zero. The eigenvalues of interest are interior eigenvalues, located in the interval $(0.0001,0.024)$. Numerical experiments with these matrices can be found, for example, in [22, Section 5.1] and [51, Example 4.4.2].

TABLE 6.7

The Atlantic Ocean model from Example 6.4 with the convergence criterion (3.6) and tol $=10^{-7}$.

\begin{tabular}{|c|c|c|c|c|c|c|c|c|c|}
\hline$m$ & \multicolumn{3}{|c|}{$k_{ \pm}=2$} & \multicolumn{3}{|c|}{$k_{ \pm}=6$} & \multicolumn{3}{|c|}{$k_{+}=0, k_{-}=6$} \\
\hline & \multicolumn{9}{|c|}{ using an inverse } \\
\hline 3 & 25 & $10^{-13}$ & $(0.30)$ & 28 & $10^{-12}$ & $(0.78)$ & 34 & $10^{-12}$ & $(0.61)$ \\
\hline 5 & 15 & $10^{-13}$ & $(0.22)$ & 21 & $10^{-12}$ & $(0.96)$ & 31 & $10^{-12}$ & $(0.76)$ \\
\hline \multirow[t]{2}{*}{10} & 15 & $10^{-12}$ & $(0.30)$ & 21 & $10^{-11}$ & $(2.88)$ & 21 & $10^{-12}$ & $(1.15)$ \\
\hline & \multicolumn{9}{|c|}{ using a decomposition } \\
\hline 3 & 15 & $10^{-10}$ & $(0.14)$ & 22 & $10^{-9}$ & $(0.40)$ & 58 & $10^{-9}$ & $(0.62)$ \\
\hline 5 & 13 & $10^{-10}$ & $(0.15)$ & $\infty$ & $10^{-1}$ & $(3.30)$ & $\infty$ & $10^{-2}$ & $(2.08)$ \\
\hline 10 & 11 & $10^{-9}$ & $(0.17)$ & $\infty$ & $10^{-2}$ & $(7.10)$ & $\infty$ & $10^{-2}$ & $(3.50)$ \\
\hline
\end{tabular}

First, we consider a finite-difference model for the shallow wave equations for the Atlantic Ocean. The corresponding matrix $A$ is of order 362 with $\lambda_{\min } \approx-3.55 \cdot 10^{-12}$ and $\lambda_{\max } \approx 0.77$. Here we want to compute the eigenpairs around the shift $\lambda_{a}=0.024$. Therefore, we apply Algorithm 3.1 to the pair $\left(I,\left(A-\lambda_{a} I\right)^{-1}\right)$ and to the pair $\left(G^{H} G, J\right)$, where 


\section{ETNA}

Kent State University and

Johann Radon Institute (RICAM)

TABLE 6.8

The Atlantic Ocean model from Example 6.4 with $m=10$ and the convergence criterion (6.7).

\begin{tabular}{c|rrrrrr} 
tol & \multicolumn{4}{|c}{$k_{ \pm}=6$} & \multicolumn{4}{c}{$k_{+}=0, k_{-}=6$} \\
\hline & \multicolumn{5}{|c}{ using an inverse } \\
\hline $10^{-7}$ & 14 & $10^{-10}$ & $(1.51)$ & 16 & $10^{-10}$ & $(0.32)$ \\
$10^{-8}$ & 16 & $10^{-11}$ & $(1.62)$ & 19 & $10^{-11}$ & $(0.40)$ \\
\hline & \multicolumn{5}{|c}{ using a decomposition } \\
\hline $10^{-7}$ & 8 & $10^{-4}$ & $(0.22)$ & 10 & $10^{-5}$ & $(0.14)$ \\
$10^{-8}$ & 11 & $10^{-5}$ & $(0.34)$ & 11 & $10^{-6}$ & $(0.16)$
\end{tabular}

$A-\lambda_{a} I=G J G^{H}$. In our implementation we have $\left\|\left(A-\lambda_{a} I\right)-G J G^{H}\right\|_{2} \approx 10^{-15}$. The results are reported in Table 6.7. For example, Algorithm 3.1 with $m=10$ applied to the matrix pair $\left(I,\left(A-\lambda_{a} I\right)^{-1}\right)$ needs 21 iterations to compute approximations for the first 6 eigenpairs on both sides of $\lambda_{a}$. The corresponding absolute error is of order $10^{-11}$ and Algorithm 3.1 runs 2.88 seconds. However, Algorithm 3.1 applied to the decomposition of $A-\lambda_{a} I$ does not converge within the allowed number of iterations. A possible way to avoid such a non-convergence is to use another convergence criterion (see the discussion in [17, Section 4]), such as

$$
\left\|r_{j}^{ \pm}\right\|_{2} \leq \text { tol } \cdot\left(\|A\|_{2}+\left|\theta_{j}^{ \pm}\right|\|B\|_{2}\right)\left\|x_{j}^{ \pm}\right\|_{2} .
$$

For large sparse matrices estimates of the 2-norms are used; in this example $B=I$. The results with $m=10$ and the new convergence criterion are reported in Table 6.8. The convergence is now achieved in all cases when using a decomposition. When applying Algorithm 3.1 to the pair $\left(I,\left(A-\lambda_{a} I\right)^{-1}\right)$, the algorithm takes fewer iterations by using the convergence criterion (6.7) than by using (3.6), but with larger absolute error. By decreasing the tolerance tol (for example, from $10^{-7}$ to $10^{-8}$ ) we can achieve a smaller absolute error.

TABLE 6.9

The Atlantic and Indian Ocean model from Example 6.4. For every fixed $m$, the first row refers to the convergence criterion (3.6) and the second row to the convergence criterion (6.7).

\begin{tabular}{|c|c|c|c|c|c|c|c|c|c|}
\hline \multirow[t]{2}{*}{$m$} & \multicolumn{3}{|c|}{$k_{ \pm}=2$} & \multicolumn{3}{|c|}{$k_{ \pm}=6$} & \multicolumn{3}{|c|}{$k \pm=20$} \\
\hline & \multicolumn{9}{|c|}{ using an inverse } \\
\hline \multirow{2}{*}{3} & 33 & $10^{-12}$ & $(0.91)$ & 41 & $10^{-12}$ & $(2.23)$ & 43 & $10^{-11}$ & $(10.89)$ \\
\hline & 20 & $10^{-10}$ & $(0.55)$ & 25 & $10^{-9}$ & $(1.31)$ & 36 & $10^{-9}$ & $(9.06)$ \\
\hline \multirow{2}{*}{5} & 24 & $10^{-13}$ & $(0.79)$ & 25 & $10^{-11}$ & $(2.58)$ & 41 & $10^{-11}$ & $(31.16)$ \\
\hline & 18 & $10^{-10}$ & $(0.49)$ & 18 & $10^{-9}$ & $(1.53)$ & 31 & $10^{-9}$ & $(23.51)$ \\
\hline \multirow{3}{*}{10} & 21 & $10^{-13}$ & $(1.06)$ & 21 & $10^{-13}$ & $(4.88)$ & 38 & $10^{-12}$ & $(133.7)$ \\
\hline & 14 & $10^{-10}$ & $(0.49)$ & 14 & $10^{-10}$ & $(2.31)$ & 27 & $10^{-9}$ & $(88.49)$ \\
\hline & \multicolumn{9}{|c|}{ using a decomposition } \\
\hline \multirow{2}{*}{3} & 21 & $10^{-9}$ & $(1.36)$ & $\infty$ & $10^{-2}$ & $(8.70)$ & $\infty$ & $10^{-1}$ & $(30.36)$ \\
\hline & 9 & $10^{-3}$ & $(0.59)$ & 12 & $10^{-4}$ & $(0.98)$ & 25 & $10^{-4}$ & $(7.15)$ \\
\hline \multirow{2}{*}{5} & $\infty$ & $10^{-2}$ & $(7.53)$ & $\infty$ & $10^{-2}$ & $(13.91)$ & $\infty$ & $10^{-1}$ & $(60.41)$ \\
\hline & 8 & $10^{-3}$ & $(0.47)$ & 9 & $10^{-4}$ & $(1.01)$ & $\infty$ & $10^{-1}$ & $(60.21)$ \\
\hline \multirow{2}{*}{10} & 21 & $10^{-9}$ & $(1.44)$ & $\infty$ & $10^{-2}$ & $(26.60)$ & $\infty$ & $10^{-2}$ & (184.7) \\
\hline & 8 & $10^{-3}$ & $(0.47)$ & 8 & $10^{-4}$ & $(0.95)$ & 11 & $10^{-4}$ & (10.04) \\
\hline
\end{tabular}




\section{ETNA}

Kent State University and

Johann Radon Institute (RICAM)

TABLE 6.10

The Laplacian eigenvalue problem on the $115 \times 115$ uniform mesh from Example 6.5. For every fixed $m$, the first row refers to the convergence criterion (3.6) and the second row to the convergence criterion (6.7).

\begin{tabular}{r|rllllllllr}
$m$ & \multicolumn{9}{c}{$k_{ \pm}=1$} & \multicolumn{9}{c}{$k_{ \pm}=5$} & \multicolumn{3}{c}{$k \pm=10$} \\
\hline & \multicolumn{1}{c}{ using an inverse } \\
\hline \multirow{2}{*}{3} & 10 & $10^{-14}$ & $(0.77)$ & 27 & $10^{-12}$ & $(7.99)$ & 41 & $10^{-10}$ & $(24.68)$ \\
& 8 & $10^{-11}$ & $(0.51)$ & 19 & $10^{-9}$ & $(4.51)$ & 33 & $10^{-9}$ & $(19.26)$ \\
\hline \multirow{2}{*}{5} & 10 & $10^{-15}$ & $(0.82)$ & 19 & $10^{-12}$ & $(9.10)$ & 41 & $10^{-10}$ & $(118.7)$ \\
& 8 & $10^{-11}$ & $(0.63)$ & 15 & $10^{-10}$ & $(5.55)$ & 22 & $10^{-9}$ & $(47.72)$ \\
\hline \multirow{2}{*}{10} & 10 & $10^{-14}$ & $(0.99)$ & 20 & $10^{-12}$ & $(21.24)$ & 21 & $10^{-11}$ & $(100.0)$ \\
& 8 & $10^{-11}$ & $(0.53)$ & 12 & $10^{-9}$ & $(10.35)$ & 15 & $10^{-10}$ & $(49.37)$ \\
\hline & & & & \multicolumn{1}{c}{ using a decomposition } & & & \\
\hline \multirow{3}{*}{3} & 9 & $10^{-11}$ & $(1.70)$ & 52 & $10^{-9}$ & $(17.81)$ & $\infty$ & $10^{-1}$ & $(64.61)$ \\
& 3 & $10^{-2}$ & $(0.62)$ & 6 & $10^{-2}$ & $(1.92)$ & 7 & $10^{-2}$ & $(3.99)$ \\
\hline \multirow{2}{*}{5} & 9 & $10^{-11}$ & $(1.89)$ & $\infty$ & $10^{-1}$ & $(55.28)$ & $\infty$ & $10^{-1}$ & $(114.5)$ \\
& 3 & $10^{-2}$ & $(0.67)$ & 41 & $10^{-2}$ & $(20.41)$ & 7 & $10^{-3}$ & $(5.28)$ \\
\hline \multirow{2}{*}{10} & 9 & $10^{-11}$ & $(1.72)$ & $\infty$ & $10^{-1}$ & $(96.29)$ & $\infty$ & $10^{-1}$ & $(265.2)$ \\
& 3 & $10^{-2}$ & $(0.56)$ & 6 & $10^{-2}$ & $(2.30)$ & 7 & $10^{-3}$ & $(5.93)$
\end{tabular}

Next, we consider a finite-difference model for the shallow wave equations for the Atlantic and Indian Ocean. The corresponding matrix $A$ is of order 1919 with $\lambda_{\min } \approx-3.40 \cdot 10^{-16}$ and $\lambda_{\max } \approx 2.92$. Here we want to compute the eigenpairs around the shift 0.0121 in the middle of the interval of interest. We have $\left\|(A-0.0121 I)-G J G^{H}\right\|_{2} \approx 10^{-14}$ in our implementation. The results are reported in Table 6.9. For $m=10$ and $k \pm=2$ by using the convergence criterion (3.6), Algorithm 3.1 applied to the inverse and Algorithm 3.1 applied to the decomposition terminate after 21 iterations, but the former is faster and more accurate than the latter. In all cases when our algorithm terminates within the allowed number of iterations, it takes fewer iterations by using the convergence criterion (6.7) than by using (3.6), but with larger absolute error. Algorithm 3.1 applied to the decomposition is quite sensitive to a convergence criterion. In many cases, it does not converge within the allowed number of iterations when using (3.6).

By observing the results from Tables 6.7-6.9, we can see that Algorithm 3.1 applied to the inverse is more accurate than Algorithm 3.1 applied to the decomposition regardless of a convergence criterion.

EXAMPLE 6.5. In this example, we consider a sparse ordinary eigenvalue problem with a real symmetric positive definite matrix. More precisely, we consider a five-point finite difference discretization of the Laplace operator on a $115 \times 115$ uniform mesh of the unit square without the circle with radius 0.5 centered in the left vertex. The corresponding matrix $A$ is of order 10,279. We want to detect first $k_{ \pm}=1,5,10$ eigenvalues around the shift $\lambda_{a}=7$. Therefore, we apply Algorithm 3.1 to the pair $\left(I,\left(A-\lambda_{a} I\right)^{-1}\right)$ and to the pair $\left(G^{H} G, J\right)$, where $A-\lambda_{a} I=G J G^{H}$. In our implementation we have $\left\|\left(A-\lambda_{a} I\right)-G J G^{H}\right\|_{2} \approx 10^{-14}$. The results are reported in Table 6.10.

As in the previous example, in all cases when our algorithm terminates within the allowed number of iterations, it takes fewer iterations by using the convergence criterion (6.7) than by using (3.6), but with larger absolute error. When using the decomposition the absolute error is satisfactory with (3.6) (in the cases when the algorithm terminated within the allowed number of iterations), but is very unsatisfactory with (6.7). In the latter case the algorithm terminates too quickly with very poor approximations of the wanted eigenpairs. 
EXAMPLE 6.6. In the last example, we consider a quadratic eigenvalue problem (6.3) with matrices $M, D, K$ from the spring example (6.6). The positive definiteness of $M, D, K$ implies both $A, B$ from (6.4) to be indefinite. Therefore, in detecting an arbitrary spectral gap we need to use the pair (5.2) or Proposition 5.4. First, we apply "perfect shuffling" [58, p. 104] to the matrix pair $(A, B)$, that is, we use auxiliary matrices $A_{1}=P^{H} A P, B_{1}=P^{H} B P$, where $P$ is a permutation matrix given by $P e_{2 j-1}=e_{j}, P e_{2 j}=e_{j+n}, j=1, \ldots, n$ and $e_{j}$ is the $j$ th column of the identity matrix $I_{2 n}$. This is a trick that transforms both matrices $A, B$ to pentadiagonal matrices $A_{1}, B_{1}$ :

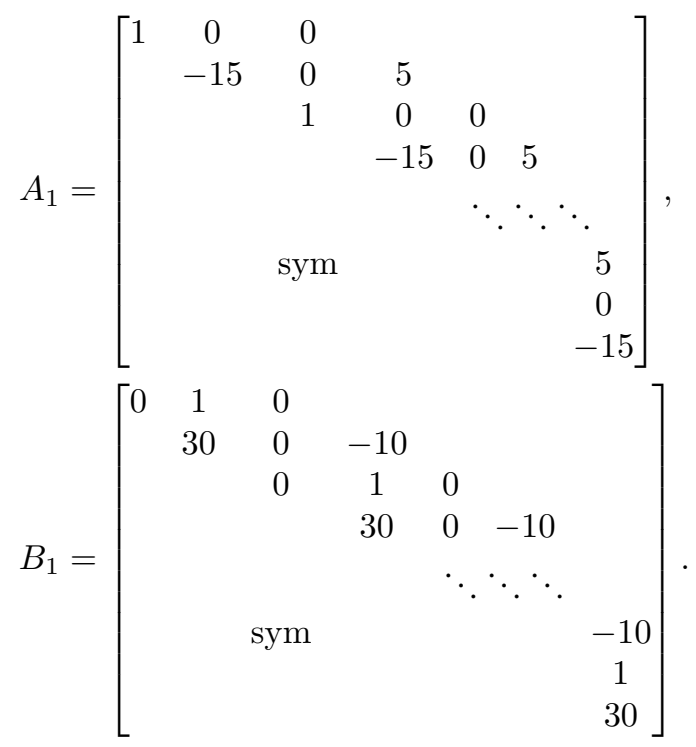

The matrix pair $(A, B)$ is congruent to $\left(A_{1}, B_{1}\right)$ : the eigenvalues are the same, and the eigenvectors are multiplied by $P$. Let $\lambda_{0}$ be an arbitrary definitizing shift and $\lambda_{a}$ the given shift from any spectral gap of the positive definite matrix pair $(A, B)$ from (6.4). Set $\widetilde{A}_{1}=A_{1}-\lambda_{0} B_{1} \succ 0$.

TABLE 6.11

QEP from Example 6.6 using inverses. For every fixed $m$, the first row refers to the convergence criterion (3.6) and the second row to the convergence criterion (6.7).

\begin{tabular}{c|lllllllll}
$m$ & \multicolumn{4}{|c}{$k_{ \pm}=1$} & \multicolumn{4}{c}{$k_{ \pm}=5$} & \multicolumn{3}{c}{$k_{ \pm}=10$} \\
\hline \multirow{2}{*}{3} & 15 & $10^{-11}$ & $(0.37)$ & 24 & $10^{-10}$ & $(0.89)$ & 28 & $10^{-10}$ & $(1.72)$ \\
& 11 & $10^{-9}$ & $(0.36)$ & 19 & $10^{-8}$ & $(0.63)$ & 22 & $10^{-8}$ & $(1.30)$ \\
\hline \multirow{2}{*}{5} & 14 & $10^{-12}$ & $(0.41)$ & 55 & $10^{-10}$ & $(4.04)$ & 25 & $10^{-9}$ & $(4.94)$ \\
& 10 & $10^{-8}$ & $(0.31)$ & 21 & $10^{-8}$ & $(1.33)$ & 21 & $10^{-8}$ & $(3.39)$ \\
\hline \multirow{2}{*}{10} & 14 & $10^{-12}$ & $(0.49)$ & 31 & $10^{-10}$ & $(7.34)$ & 30 & $10^{-11}$ & $(18.2)$ \\
& 12 & $10^{-9}$ & $(0.39)$ & 19 & $10^{-8}$ & $(3.91)$ & 17 & $10^{-8}$ & $(8.70)$
\end{tabular}

Consider the indefinite decomposition $\widetilde{B}_{1}=B_{1}-\left(\lambda_{a}-\lambda_{0}\right)^{-1} \widetilde{A}_{1}=C J C^{H}$ obtained by [54], but with a pivoting strategy given in [12, Algorithm E]. That pivoting strategy is designed for pentadiagonal real symmetric or Hermitian matrices and preserves the pentadiagonal structure during the process. In our implementation we have $\left\|\widetilde{B}_{1}-C J C^{H}\right\|_{2} \approx 10^{-14}$. Let $L$ be the Cholesky factor of $\widetilde{A}_{1}$; cf. Theorem 5.4. Set $n=1000$ in (6.6) and $\lambda_{0}=-5$. We want to detect first $k_{ \pm}=1,5,10$ eigenvalues around the shift $\lambda_{a}=-30$; in the middle 


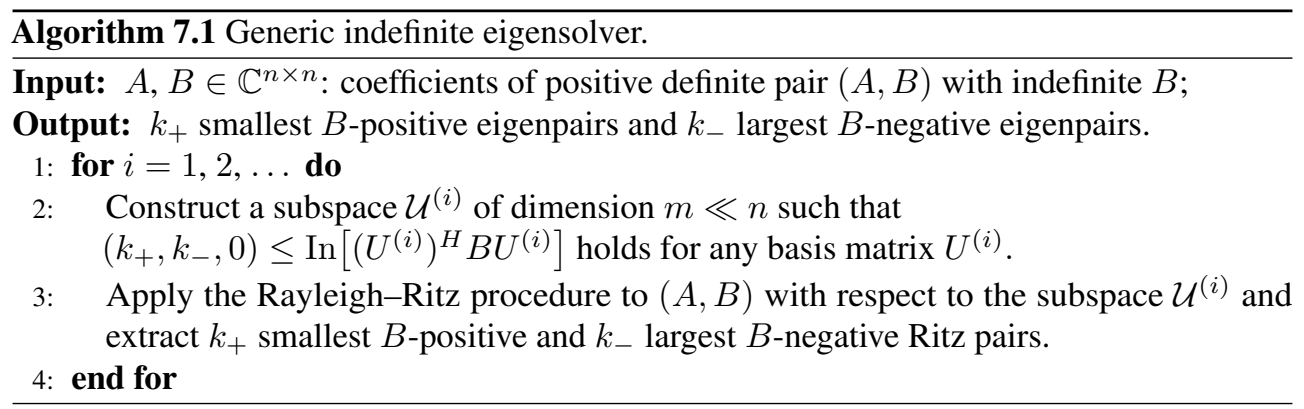

of $B$-negative eigenvalues: see Figure 6.1. Therefore, we apply Algorithm 3.1 to the pair $\left(\widetilde{A}_{1}^{-1}, \widetilde{B}_{1}^{-1}\right)$ and to the pair $\left(C^{H} C, J\right)$. The results are reported in Table 6.11 . We omit the results when using decompositions since, in almost all cases, either the algorithm does not converge within the allowed number of iterations or the absolute error is $O\left(10^{-3}\right)$ or larger.

7. Conclusions. Numerical experiments [35] for a generalized eigenvalue problem with both symmetric positive definite matrices demonstrate that a $(k)$-scheme of preconditioned gradient iterations $[44,45]$ for $k \geq 4$ is of minor importance and that the locally optimal block preconditioned conjugate gradient (LOBPCG) iteration is an optimal eigensolver within that $(k)$-scheme. In our experiments, we noticed that indefinite LOBPCG is much faster than indefinite block preconditioned steepest descent/ascent (BPSD/A). A further increase in the dimension of the search subspaces often leads to a decrease in the total number of iterations of our Algorithm 3.1; it is especially significant when a preconditioner is poor (when a definitizing shift is not close to the boundaries of the definiteness interval), although it is more memory- and time-consuming. When excellent preconditioners are used, we can conclude that indefinite LOBPCG is an optimal eigensolver within our indefinite $(m)$-scheme, that is, Algorithm 3.1. In some cases, very crude approximations of the preconditioned residuals can be used.

There is an important difference between LOBPCG type algorithms designed for a hyperbolic quadratic eigenvalue problem $[7,41,56]$ and our indefinite LOBPCG type algorithm applied to the matrix pair from the linearization: the former can use any initial guess, the latter needs to contain at least as many $B$-positive and $B$-negative vectors as we want to compute. Many new indefinite eigensolvers can be proposed using some appropriate search subspace and applying the Rayleigh-Ritz projection method that extracts interior eigenpairs. The generic indefinite algorithm (cf. [51, Algorithm 2.1]) is given in Algorithm 7.1. If the column space of every iteration matrix of the Ritz vectors is included in the subspace $\mathcal{U}^{(i)}$, then choosing a valid initial guess will provide enough $B$-positive and $B$-negative vectors in any basis matrix $U^{(i)}$ of $\mathcal{U}^{(i)}$ for all $i=1,2, \ldots$. Some choices of the subspace $\mathcal{U}^{(i)}$ of Algorithm 7.1 can, with a good separation of the desired eigenvalues, lead to the fast convergence without preconditioning. However, if preconditioning is used, then we propose to add the column space of the preconditioned residual of the current iteration matrix to the subspace $\mathcal{U}^{(i)}$. The crucial thing is to know at least one definitizing shift; it is trivial if $A$ is positive definite. For finding such a shift, we can use the algorithms given in [23] or [43, Section 2.2]. For some examples in applications, knowing only one definitizing shift is not enough, two are necessary. Not just any two definitizing shifts, but those shifts that are close to the boundaries of the definiteness interval. Finding an algorithm to compute two such definitizing shifts is a matter for future work.

Finally, we derived some ideas on how to use an indefinite eigensolver (for example, Algorithm 3.1) to compute a few eigenvalues around any spectral gap and the corresponding 
eigenvectors of a definite matrix pair. The proposed spectral transformations can be applied to those definite matrix pairs for which solving the corresponding linear systems is easy or performing decompositions is inexpensive and can be done very accurately. Our experiments demonstrate that the application of Algorithm 3.1 to a transformed pair with inverses is more efficient than the application to a transformed pair with decompositions. Enlarging the dimension of the search subspaces can reduce the total number of iterations of Algorithm 3.1, but often increases CPU time. Therefore, we can recommend to use $m=3$ in our indefinite $m$-scheme, that is, to use the indefinite LOBPCG iteration.

Acknowledgments. Some basic ideas of this paper emerged during the author's study visit to the ANCHP, MATHICSE, EPF Lausanne, Switzerland, under the direction of Professor Daniel Kressner to whom the author is indebted for his illuminating discussions and comments, as well as for kind hospitality. The author also thanks Dr. Meiyue Shao for all the patience and support during the formation of paper [38], the predecessor of this paper, and for the discussion of $B$-orthonormality. The author thanks Professors Krešimir Veselić and Ninoslav Truhar for valuable discussions and Professor Klaus Neymeyr for clarifying some details in the proof of [46, Theorem 2.2]. The author thanks the editor and the anonymous referees for many detailed and valuable comments and suggestions.

\section{REFERENCES}

[1] C. Ashcraft, R. G. Grimes, And J. G. Lewis, Accurate symmetric indefinite linear equation solvers, SIAM J. Matrix Anal. Appl., 20 (1999), pp. 513-561.

[2] Z. Bai, J. Demmel, J. DongarRa, A. Ruhe, And H. VAn Der Vorst, eds., Templates for the Solution of Algebraic Eigenvalue Problems: A Practical Guide, SIAM, Philadelphia, 2000.

[3] Z. BAI AND R.-C. LI, Minimization principles for the linear response eigenvalue problem I: Theory, SIAM J. Matrix Anal. Appl., 33 (2012), pp. 1075-1100.

[4] - Minimization principles for the linear response eigenvalue problem II: Computation, SIAM J. Matrix Anal. Appl., 34 (2013), pp. 392-416.

[5] - Minimization principles and computation for the generalized linear response eigenvalue problem, BIT, 54 (2014), pp. 31-54.

[6] Z. BAI, R. LI, AND W. LIN, Linear response eigenvalue problem solved by extended locally optimal preconditioned conjugate gradient methods, Sci. China Math., 59 (2016), pp. 1443-1460.

[7] P. Benner AND X. LIANG, Convergence analysis of extended LOBPCG for the extreme eigenvalue, in preparation.

[8] T. Betcke, N. J. Higham, V. Mehrmann, C. Schröder, and F. Tisseur, NLEVP: a collection of nonlinear eigenvalue problems, ACM Trans. Math. Software, 39 (2013), Art. 7 (28 pages). https://github.com/ftisseur/nlevp

[9] R. Boisvert, R. Pozo, K. Remington, B. Miller, And R. Lipman, Matrix Market, National Institute of Standards and Technology, 1996. Available at http://math.nist.gov/MatrixMarket/

[10] J. R. BUNCH, Analysis of the diagonal pivoting method, SIAM J. Numer. Anal., 8 (1971), pp. 656-680.

[11] - Partial pivoting strategies for symmetric matrices, SIAM J. Numer. Anal., 11 (1974), pp. 521-528.

[12] J. R. BUNCH AND L. KAUFMAN, Some stable methods for calculating inertia and solving symmetric linear systems, Math. Comp., 31 (1977), pp. 163-179.

[13] J. R. Bunch, L. Kaufman, And B. N. Parlett, Decomposition of a symmetric matrix, Numer. Math., 27 (1976), pp. 95-109.

[14] J. R. BUNCH AND R. F. MARCIA, A simplified pivoting strategy for symmetric tridiagonal matrices, Numer. Linear Algebra Appl., 13 (2006), pp. 865-867.

[15] J. R. BUNCH AND B. N. PARLETT, Direct methods for solving symmetric indefinite systems of linear equations, SIAM J. Numer. Anal., 8 (1971), pp. 639-655.

[16] J. W. Demmel, Applied Numerical Linear Algebra, SIAM, Philadelphia, 1997.

[17] J. A. Duersch, M. ShaO, C. YANG, AND M. Gu, A robust and efficient implementation of LOBPCG, SIAM J. Sci. Comput., 40 (2018), pp. C655-C676.

[18] I. DufF, R. G. GRIMES, AND J. G. LEwIS, Users' guide for the Harwell-Boeing sparse matrix collection, Technical report TR/PA/92/86, CERFACS, 1992.

[19] E. G. D’yAKONOv, Optimization in Solving Elliptic Problems, CRC Press, Boca Raton, 1996. 
[20] E. G. D'yAKONOV AND M. Y. OREKHOV, Minimization of the computational labor in determining the first eigenvalues of differential operators, Math. Notes, 27 (1980), pp. 795-812.

[21] G. H. Golub AND Q. YE, An inverse free preconditioned Krylov subspace method for symmetric generalized eigenvalue problems, SIAM J. Sci. Comput., 24 (2002), pp. 312-334.

[22] R. G. GRImes, J. G. LEWIS, AND H. D. Simon, A shifted block Lanczos algorithm for solving sparse symmetric generalized eigenproblems, SIAM J. Matrix Anal. Appl., 15 (1994), pp. 228-272.

[23] C.-H. Guo, N. J. Higham, AND F. Tisseur, An improved arc algorithm for detecting definite Hermitian pairs, SIAM J. Matrix Anal. Appl., 31 (2009), pp. 1131-1151.

[24] V. HARI, S. Singer, AND S. SINGER, Block-oriented J-Jacobi methods for Hermitian matrices, Linear Algebra Appl., 433 (2010), pp. 1491-1512.

[25] U. Hetmaniuk And R. LehoucQ, Basis selection in LOBPCG, J. Comput. Phys., 218 (2006), pp. 324-332.

[26] N. J. Higham, F. Tisseur, AND P. M. VAN Dooren, Detecting a definite Hermitian pair and a hyperbolic or elliptic quadratic eigenvalue problem, and associated nearness problems, Linear Algebra Appl., 351/352 (2002), pp. 455-474.

[27] HSL, HSL: A collection of Fortran codes for large scale scientific computation, 2011. http://www.hsl.rl.ac.uk.

[28] L. KAUFMAn, The retraction algorithm for factoring banded symmetric matrices, Numer. Linear Algebra Appl., 14 (2007), pp. 237-254.

[29] A. V. KNYAZEV, Computation of Eigenvalues and Eigenvectors for Mesh Problems: Algorithms and Error Estimates, Dept. Numer. Math, USSR Acad. Sci., Moscow, 1986.

[30] - Convergence rate estimates for iterative methods for a mesh symmetric eigenvalue problem, Soviet J. Numer. Anal. Math. Modelling, 2 (1987), pp. 371-396.

[31] - A preconditioned conjugate gradient method for eigenvalue problems and its implementation in a subspace, in Numerical Treatment of Eigenvalue Problems. Vol. 5., J. Albrecht, L. Collatz, P. Hagedorn and W. Velte, eds., vol. 96 of Internat. Ser. Numer. Math., Birkhäuser, Basel, 1991, pp. 143-154.

[32] - Preconditioned eigensolvers-an oxymoron?, Electron. Trans. Numer. Anal., 7 (1998), pp. 104-123. http://etna.ricam.oeaw.ac.at/vol.7.1998/pp104-123.dir/pp104-123.pdf

[33] - Toward the optimal preconditioned eigensolver: locally optimal block preconditioned conjugate gradient method, SIAM J. Sci. Comput., 23 (2001), pp. 517-541.

[34] A. V. Knyazev, M. E. Argentati, I. Lashuk, and E. E. Ovtchinnikov, Block locally optimal preconditioned eigenvalue xolvers (BLOPEX) in hypre and PETSc, SIAM J. Sci. Comput., 29 (2007), pp. 2224-2239.

[35] A. V. KNYAZEV AND K. NEYMEYR, Efficient solution of symmetric eigenvalue problems using multigrid preconditioners in the locally optimal block conjugate gradient method, Electron. Trans. Numer. Anal., 15 (2003), pp. 38-55.

http://etna.ricam.oeaw.ac.at/vol.15.2003/pp38-55.dir/pp38-55.pdf

[36] - Gradient flow approach to geometric convergence analysis of preconditioned eigensolvers, SIAM J. Matrix Anal. Appl., 31 (2009), pp. 621-628.

[37] J. KOVAČ-STRIKO AND K. VESElić, Trace minimization and definiteness of symmetric pencils, Linear Algebra Appl., 216 (1995), pp. 139-158.

[38] D. Kressner, M. MiloloŽA Pandur, And M. Shao, An indefinite variant of LOBPCG for definite matrix pencils, Numer. Algorithms, 66 (2014), pp. 681-703.

[39] P. LANCASTER AND L. RoDMAN, Canonical forms for Hermitian matrix pairs under strict equivalence and congruence, SIAM Rev., 47 (2005), pp. 407-443.

[40] P. LANCASTER AND Q. YE, Variational and numerical methods for symmetric matrix pencils, Bull. Austral. Math. Soc., 43 (1991), pp. 1-17.

[41] X. LIANG AND R.-C. LI, The hyperbolic quadratic eigenvalue problem, Forum Math. Sigma, 3 (2015), pp. e13, 93.

[42] X. LIANG, R.-C. LI, AND Z. BAI, Trace minimization principles for positive semi-definite pencils, Linear Algebra Appl., 438 (2013), pp. 3085-3106.

[43] M. MiloložA PAndur, Computing Interior Eigenvalues and Corresponding Eigenvectors of Definite Matrix Pairs, PhD. Thesis, Faculty of Science, Department of Mathematics, University of Zagreb, 2016.

[44] K. NEYMEYR, A Hierarchy of Preconditioned Eigensolvers for Elliptic Differential Operators, Hab. Thesis, Mathematisches Institut, Universität Tübingen, 2001.

[45] - On preconditioned eigensolvers and invert-Lanczos processes, Linear Algebra Appl., 430 (2009), pp. 1039-1056.

[46] - A geometric convergence theory for the preconditioned steepest descent iteration, SIAM J. Numer. Anal., 50 (2012), pp. 3188-3207.

[47] K. Neymeyr, E. Ovtchinnikov, And M. ZHOU, Convergence analysis of gradient iterations for the symmetric eigenvalue problem, SIAM J. Matrix Anal. Appl., 32 (2011), pp. 443-456. 


\section{ETNA}

Kent State University and

Johann Radon Institute (RICAM)

[48] K. Neymeyr And M. Zhou, The block preconditioned steepest descent iteration for elliptic operator eigenvalue problems, Electron. Trans. Numer. Anal., 41 (2014), pp. 93-108.

http://etna.ricam.oeaw.ac.at/vol.41.2014/pp93-108.dir/pp93-108.pdf

[49] B. N. PARLETt, The Symmetric Eigenvalue Problem, SIAM, Philadelphia, 1998.

[50] B. T. PolyaK, Introduction to Optimization, Optimization Software, Inc., New York, 1987.

[51] P. D. QuILlEn, Generalizations of an Inverse Free Krylov Subspace Method for the Symmetric Generalized Eigenvalue Problem, PhD. Thesis, Department of Mathematics, University of Kentucky, Lexington, 2005.

[52] P. QUILLEN AND Q. YE, A block inverse-free preconditioned Krylov subspace method for symmetric generalized eigenvalue problems, J. Comput. Appl. Math., 233 (2010), pp. 1298-1313.

[53] D. RocCA, Z. BAI, R.-C. LI, AND G. GALLI, A block variational procedure for the iterative diagonalization of non-Hermitian random-phase approximation matrices, J. Chem. Phys., 136 (2012), Art. 034111 (8 pages).

[54] I. SLAPNIČAR, Componentwise analysis of direct factorization of real symmetric and Hermitian matrices, Linear Algebra Appl., 272 (1998), pp. 227-275.

[55] G. W. Stewart And J. G. Sun, Matrix Perturbation Theory, Academic Press, Boston, 1990.

[56] D. B. SZYLD AND F. XUE, Preconditioned eigensolvers for large-scale nonlinear Hermitian eigenproblems with variational characterizations. I. Extreme eigenvalues, Math. Comp., 85 (2016), pp. 2887-2918.

[57] K. VeSELIĆ, A Jacobi eigenreduction algorithm for definite matrix pairs, Numer. Math., 64 (1993), pp. 241269.

[58] K. Veselić, Damped Oscillations of Linear Systems. A Mathematical Introduction, Springer, Heidelberg, 2011. 